\title{
Design Flash Memory Programmer Device
}

\section{Dhuha Basheer Abdullah \\ Reyath Z .Mahmood}

Prof.dhuha_basheer@uomosul.edu.iq

College of Computer Sciences and Mathematics

University of Mosul, Iraq

Received on: 06/04/2005

Accepted on: 09/08/2005

\section{ABSTRACT}

The purpose of this research is to design a flash memory programmer device consisting of a set of Integrated Circuits (ICs) to meet the needs. These circuits have been connected according to operations (defining, reading, programming and erasing) run on the memory chip.

The research also deals with building a program which programs two types of Flash Memory (Multi-purpose and Bulk-Erase) by using the above mentioned hardware design. The program provides the possibility reading these two types of Flash Memory, Programming and Erasing them. The design of a Flash Memory Programmer Device and the following algorithms particular for its programming have led to a complete system regarding the Hardware and Software.

Keywords: Flash Memory, Integrated Circuits, Bulk-Erase

$$
\begin{aligned}
& \text { تصميم جهاز مبرمج الأكرة الومضية }
\end{aligned}
$$

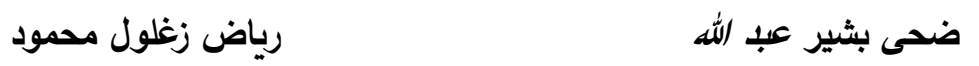

$$
\begin{aligned}
& \text { كلية علوم الحاسوب والرياضيات، جامعة الموصل عليل }
\end{aligned}
$$

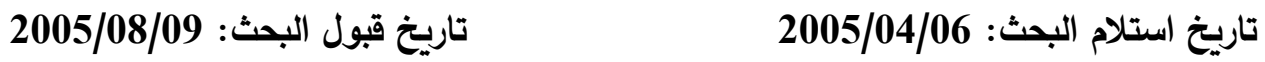

$$
\begin{aligned}
& \text { الملخص } \\
& \text { إن الغرض من هذا البحث هو تصميم جهاز مبرمج الذاكرة الومضية اذ يتكون الجهاز من }
\end{aligned}
$$

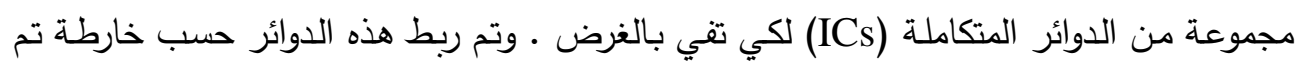

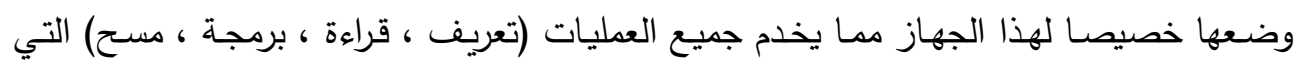

$$
\begin{aligned}
& \text { تجرى على شريحة الذاكرة . } \\
& \text { كما تتاول البحث بناء برنامج يقوم ببرمجة نوعين من الذاكرة الومضية (متعددة الأغراض ، ذات }
\end{aligned}
$$

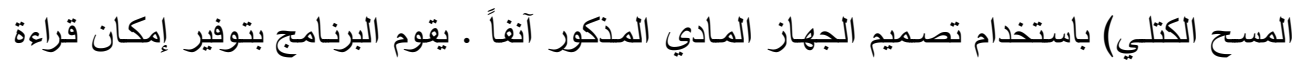

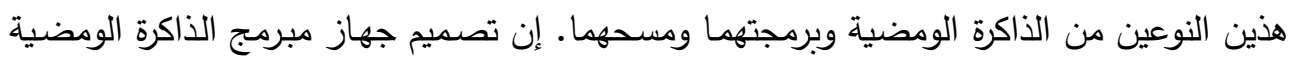

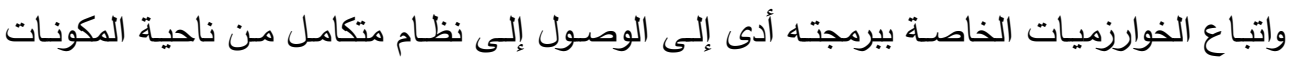

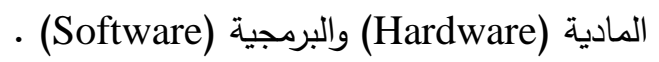


الكلمات المفتاحية: الذاكرة الومضية، الدوائر المتكاملة، المسح الكتلي.

1- المقدمة

إن الذاكرة الومضية (Flash Memory) هي ذاكرة قراءة فقط من عائلة (CMOS)

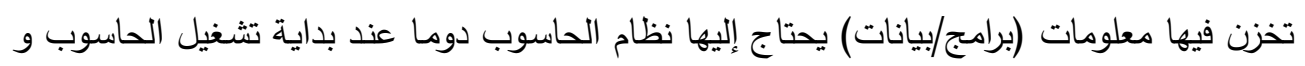

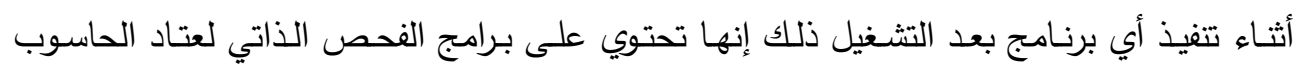
(POST)

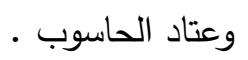

تعد الذاكرة الومضية نوعا من ذاكرات الـ EEPROM غير ان معلوماتها يمكن ان تمسح

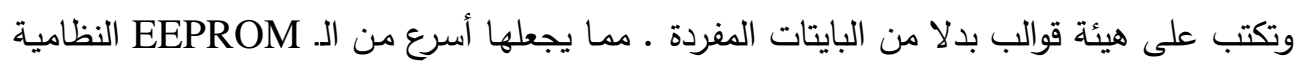

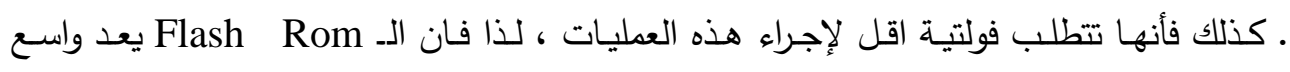
الاستخدام الآن في حفظ معلومـات الـ.BIOS الخاصـة بالحواسيب الثخصـية] [2]. وهـذا يمـنح مصنعي الـ BIOS الفرصـة لتوفير مـا يستجد عن طريق الانترنيت ويوفر للمستخدمين إمكان

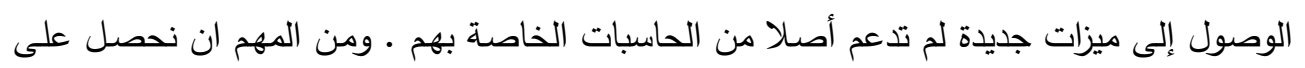

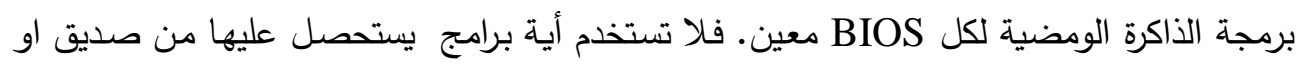

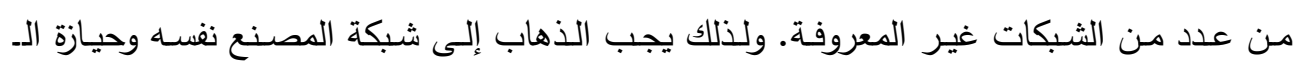

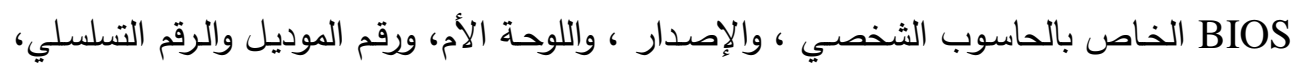

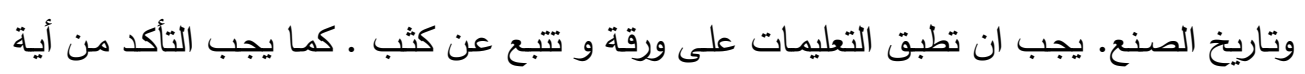

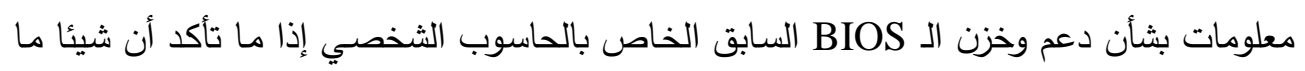

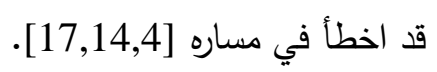
إن تقانة هذا النوع من الذاكرات مهمة في دراسة أنظمة ألحوا سيب الدقيقة ؛ فأجهزة الذاكرة

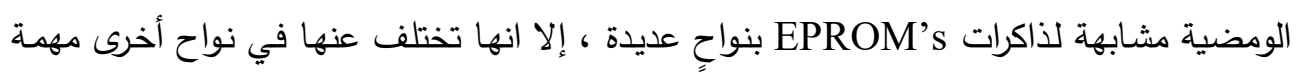

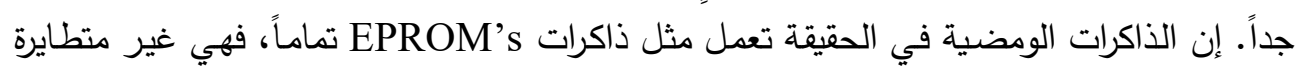
وتقرأ مثل الـ EPROM تماما وتبرمج بخوارزمية مشابهة للخوارزمية الخاصة ببرمجة الـ EPROM

أن الاختلاف الرئيس بين الذاكرة الومضية وذاكرة الـ EPROM هو ان خلايـا ذاكرتها تمسح كهربائيا ، بدلا من تعريضها لضوه الأشعة فوق البنفجية ـ أي ان مصفوفة الخزن للذاكرة

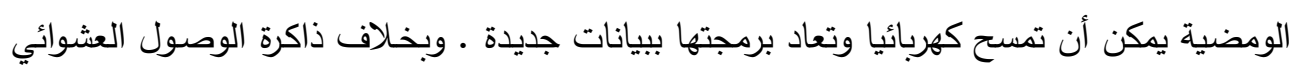

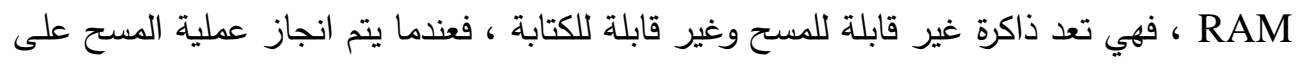


الذاكرة الومضية يتم مسح مصفوفة الذاكرة كليا أو لمقطع كبير من مواقع الخزن و ليس مسح بايت

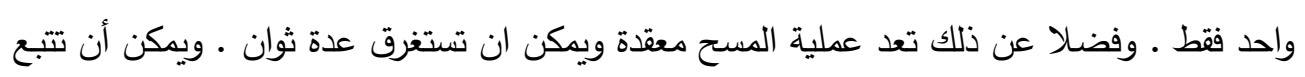

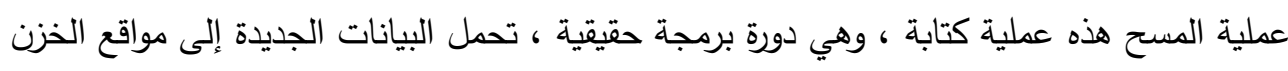

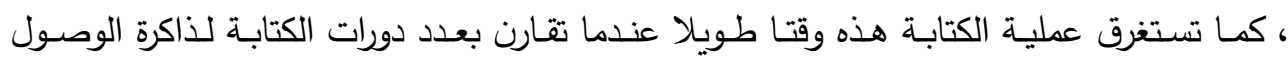

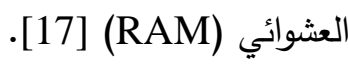

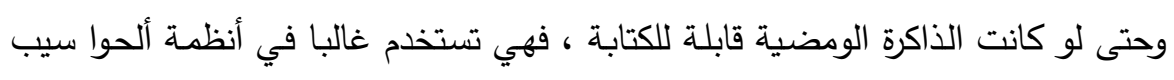

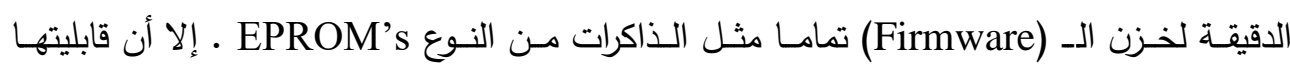

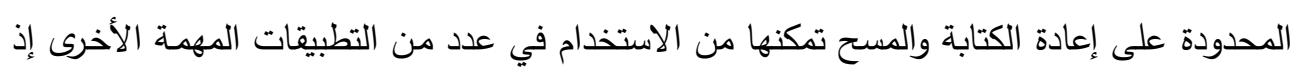
تبتغي إعادة كتابة البيانات، ولكن ليس على نحو متكرر [9,3]

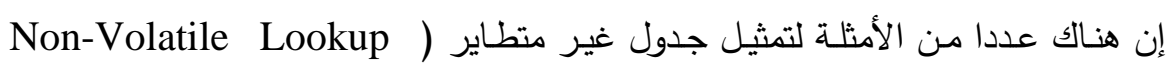

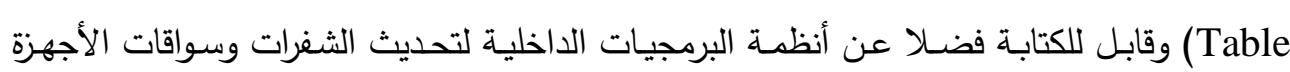
الصلبة (Solid State Drives) (9,8,7) إن الذاكرات الومضية تعمل بطريقة مشابهة لذاكرة الـ 6]EPROM] ـ ويظهر الثكل (1)

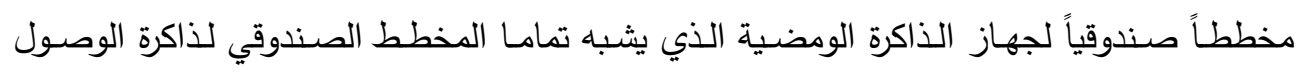

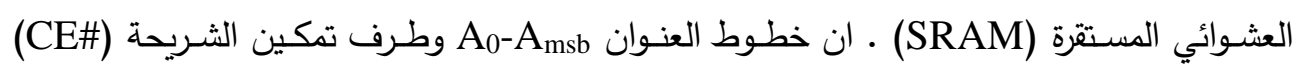

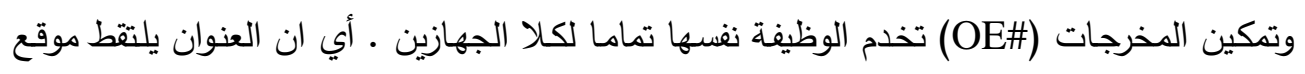

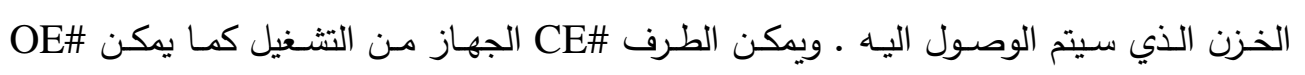

$$
\text { وصول البيانات إلى المخرجات خلال دورات القراءة . }
$$

كذلك نرى انهما يختلفان في ناحيتين: الأولى ، تعرف خطوط البيانات في الذاكرة الومضية

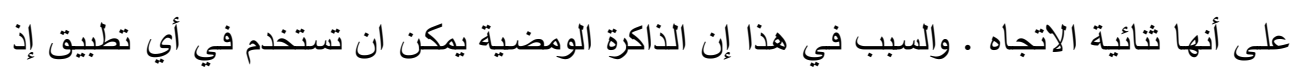

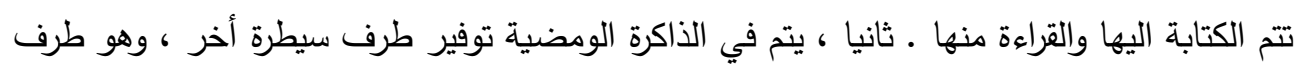

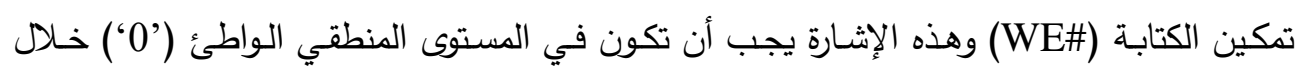

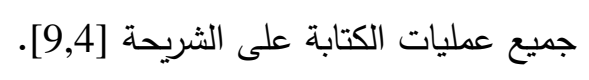




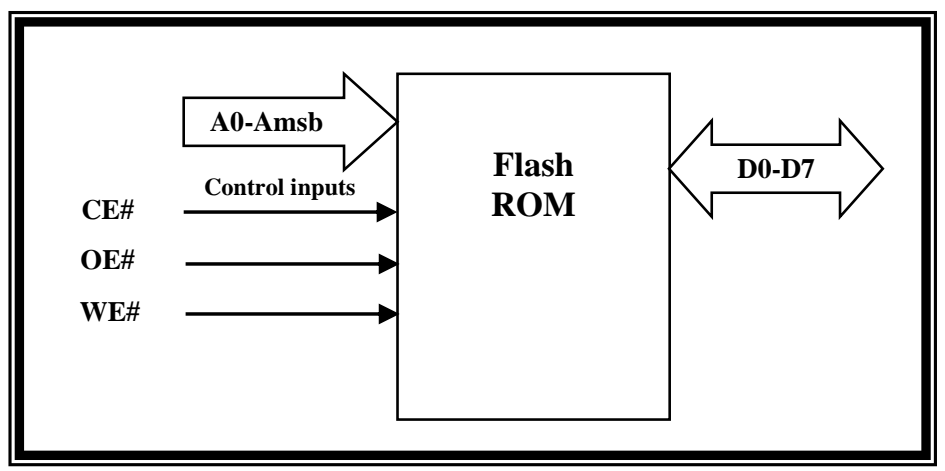

الثكل (1) : المخطط الصندوقي لذاكرة (Flash ROM)

تتوافر أجهزة الذاكرة الومضية مع معمارية المصفوفة وهي مختلفة وعديدة ؛ اذ ترتبط هذه

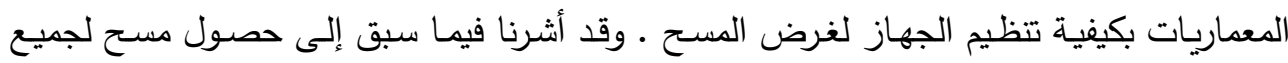
محتويات الذاكرة او لقالب كبير من مواقع خزن الذاكرة عندما يتم إنجاز عملية المسح على لفئ الذاكرة

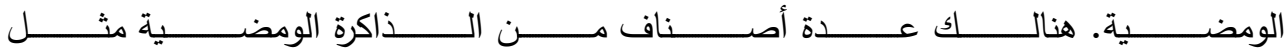
(ذاكرة المسح الكتلي Bulk-Erase وذاكرة قالب الإقلاع Boot-Block وذاكرة الملف الومضي

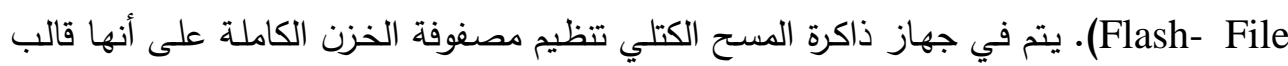

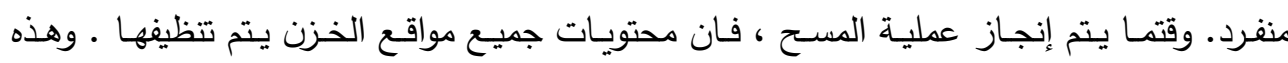

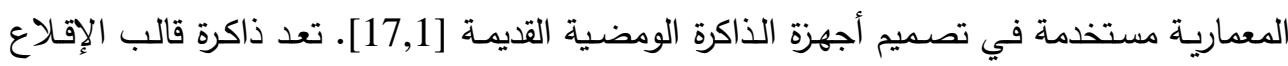

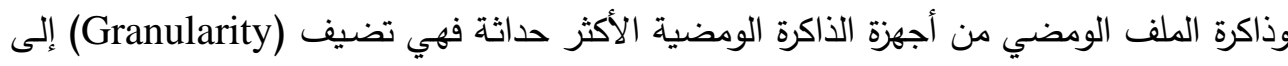
عملية البرمجة ـ ولا ينبغي لمصفوفة الذاكرة أن تمسح بالكامل ، بل يمسح كل من القوالب المستقلة

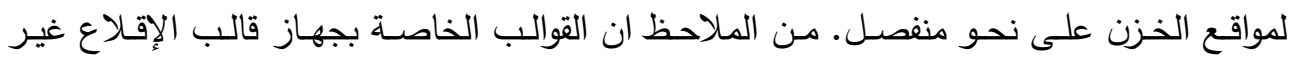

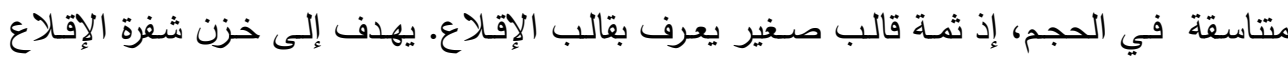

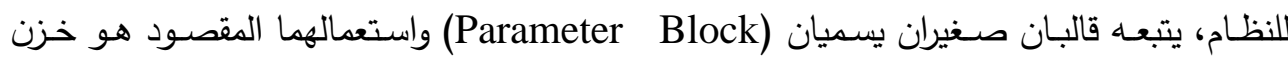

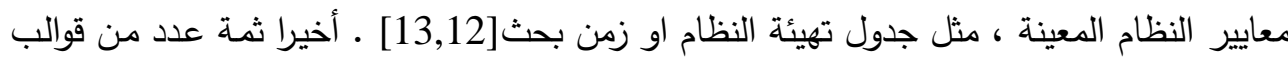

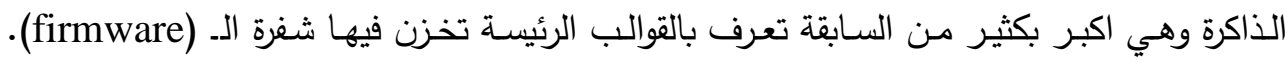
تستخدم أجهزة قالب الإقلاع في مجموعة من التطبيقات التي تتطلب سعة ذاكرة اصغر او تستقيد

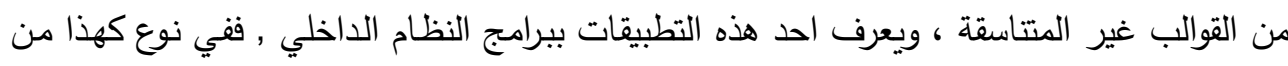


التطبيق يتم تثبيت شفرة الصندوق المستخدمة لبدء الحاسوب الدقيق في جزء قالب الإقلاع للذاكرة

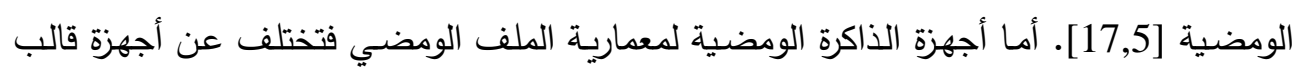
الإقلاع في كون مصفوفة الذاكرة مقسمة إلى قوالب ذات أحجام متساوية ، ولهذا السبب فانها مرتبة

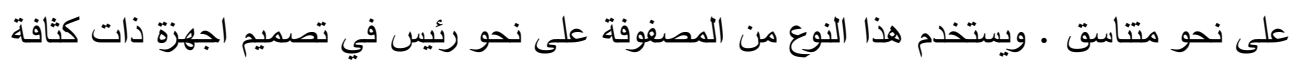

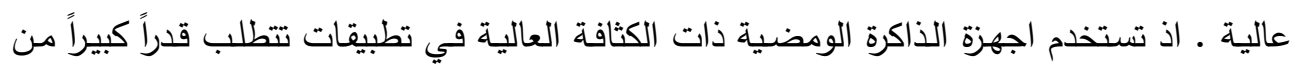

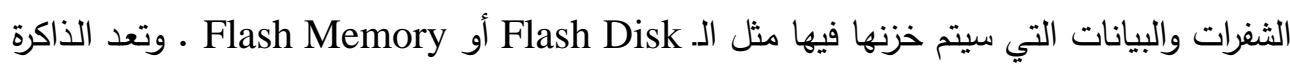

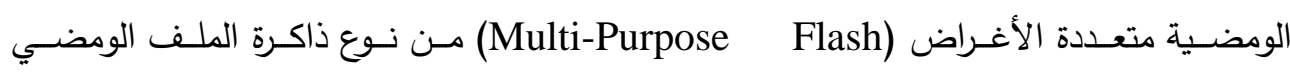
$\cdot[17,16,11,10]$

\section{2- الوصف المادي لجهاز مبرمج الذاكرة الومضية}

تم تصميم جهاز مبرمج يقوم ببرمجة مختلف أنواع ذاكرات القراءة فقط وفي مقدمتها الذاكرة

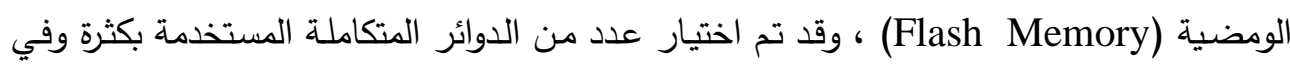

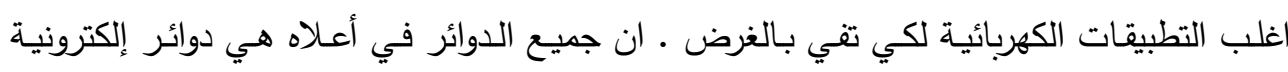

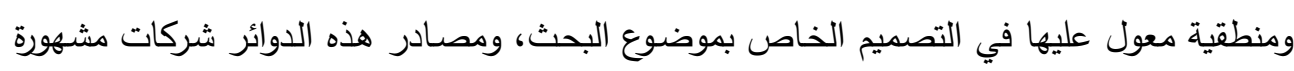

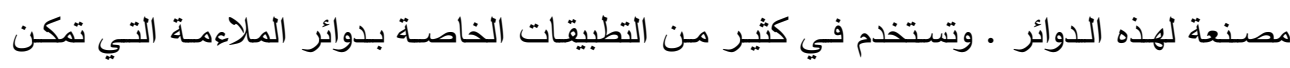

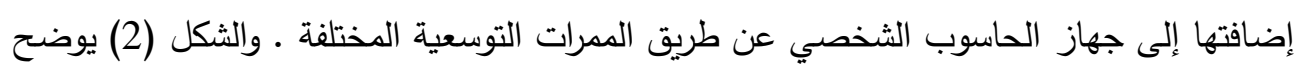
مخطط الربط لجهاز مبرمج الذاكرة الومضية.

\section{1-2استخدام منفذ الطابعة المتوازي}

تم استخدام منفذ الطابعة المتوازي لكي يربط بالجهاز المبردج عن طريق كابل البيانات

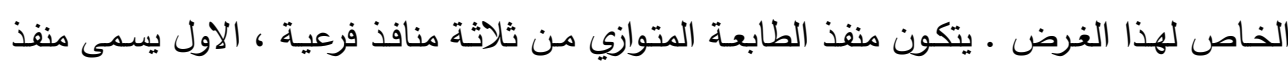

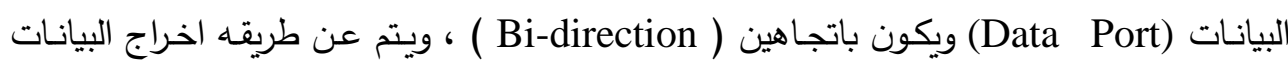

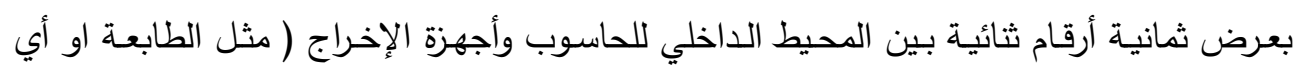

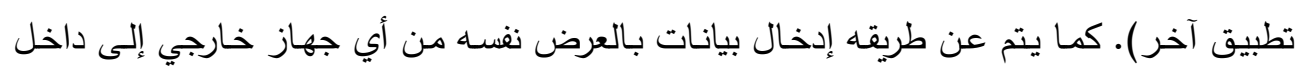

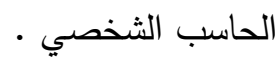
أما المنفذ الثاني فيسمى منفذ الأوامر (Command Port) ويستخدم عادة لإرسال الأوامر الخاصة بالطباعة إلى جهاز الطابعة المتصل بالحاسوب الثخصي ، وقد تم استخدامه في التصميم

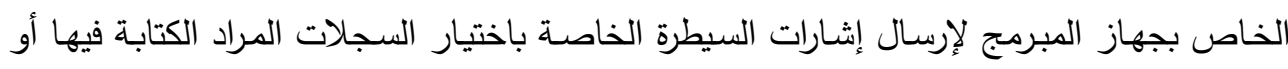


القراءة منها ، والمبنية ضمن اللوحة الخاصـة بالجهاز المبرمج وقد تم استخدام جميع إخراجات هذا

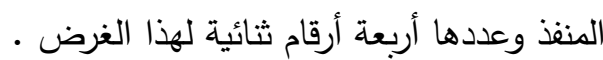

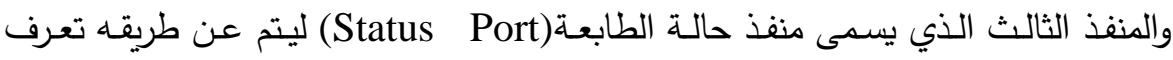

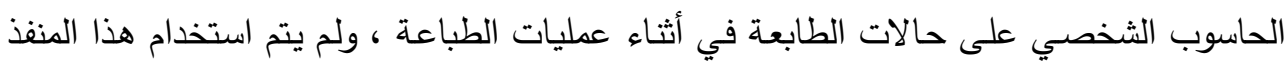

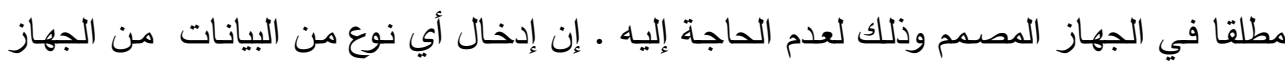

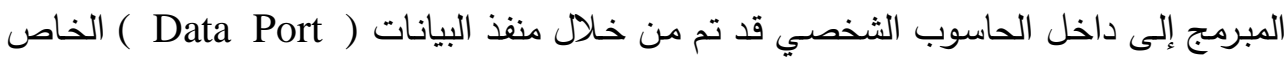
بمنفذ الطابعة المتوازي [15]. 


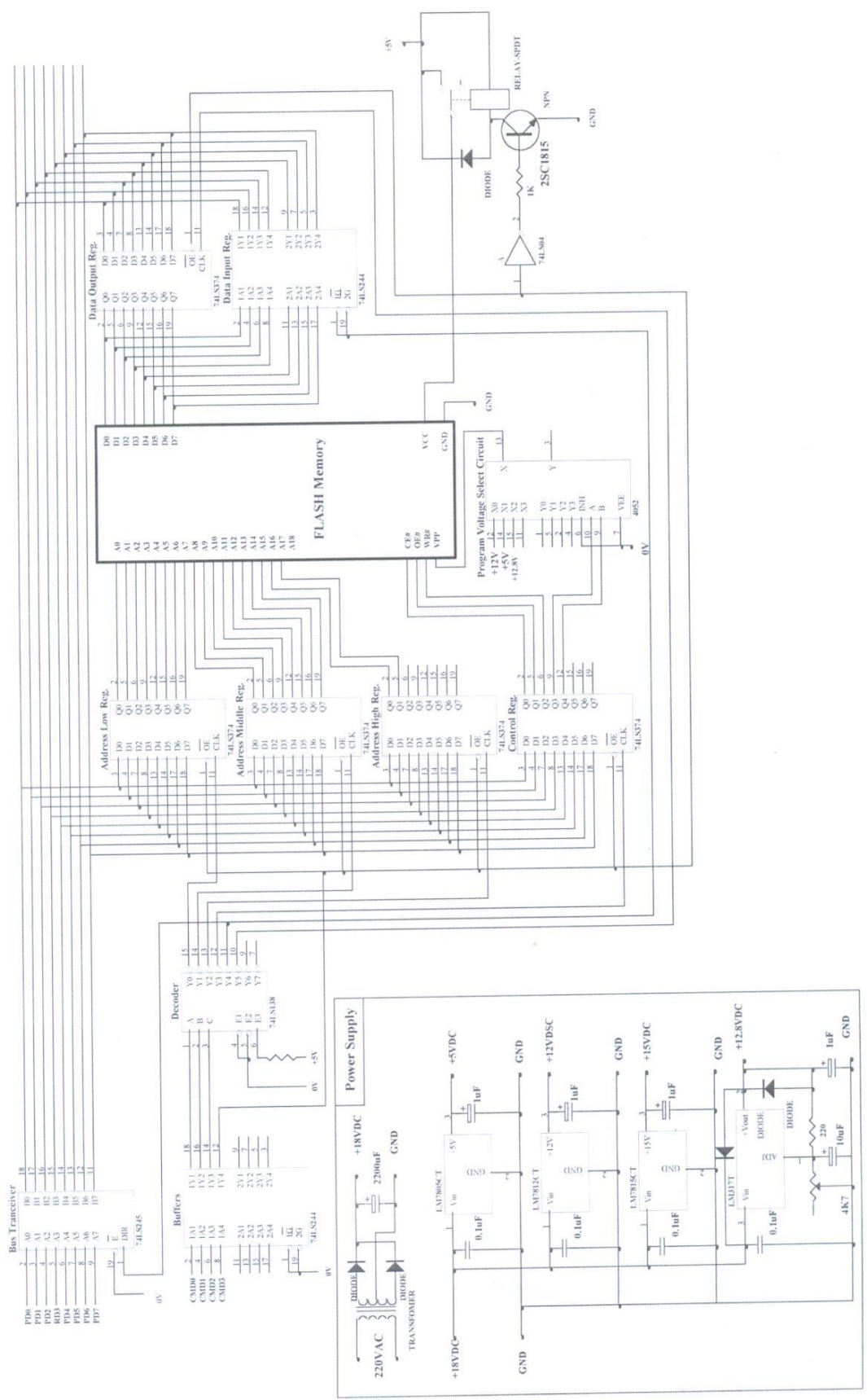

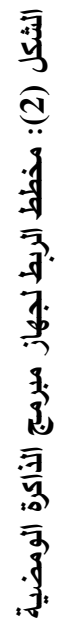




\section{2-2 2-2 المكونات المادية لجهاز مبرمج الذاكرة الومضية}

1-2-2 مجهز القدرة

220V AC يتكون مجهز القدرة من محولة خافضـة تقوم بتخفيض الفولتية المتناوبـة من

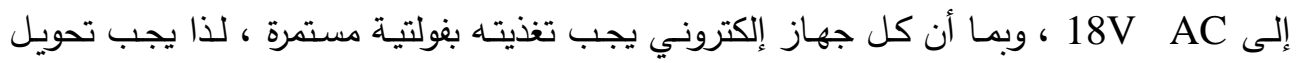
الفولتية الخارجة من المحولة من الفولتية المتتاوبة إلى الفولتية المستمرة ، وذلك باستخدام موحدات

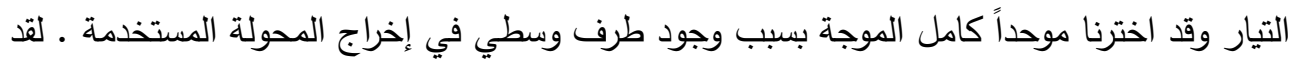
تم ربط أطراف المحولة الثلاثة بهذا الموحد المتكون من ثنائيين بلوريين إذ يمرر الأول الفولتيـة

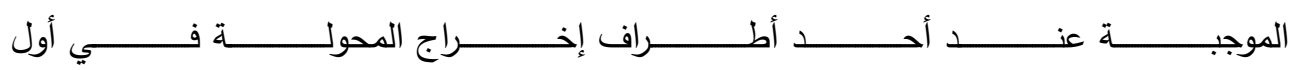
180º من الطور ، أما الثاني فيقوم بتمرير الفولتية الموجبة في الجزء الثاني من الطور • بعد ذلك نحصل على فولتية مستمرة قدرها 18V DC ولكنها غير مرشحة لذا يجب ربط إخراج الموحد

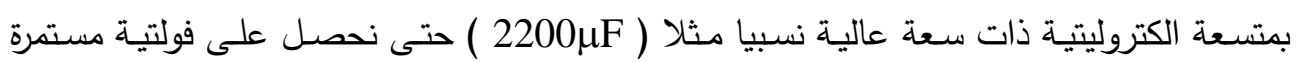
قريبة جدا من فولتية البطارية ـ ويمكن الإثارة هنا إلى أنه كلما زادت سعة المتسعة أقترب التيار

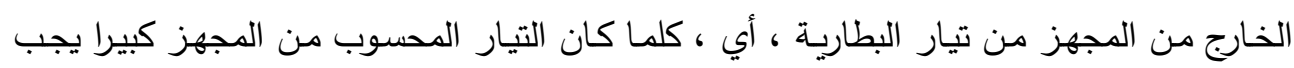

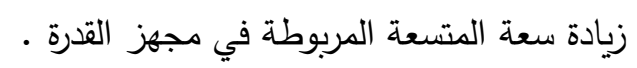

2-2-2 منظمات الفولتية

تم استخدام عدة منظمات للفولتية لتجهيز مكونات الجهاز المبرمج بالفولتيات المناسبة لكل

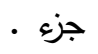

\section{* منظم الفولتية}

تم استخدام دوائر متكاملة من عائلة Transistor-Transistor-Logic ( TTL ) وهذه العائلة يجب تغذيتها بفولتية 5V 5 + نسبة سماح 5\% 5 ، لذا تم استخدام منظم الفولتية الثابت ذي مني

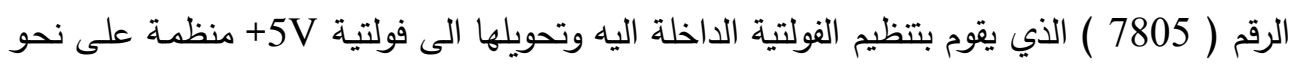

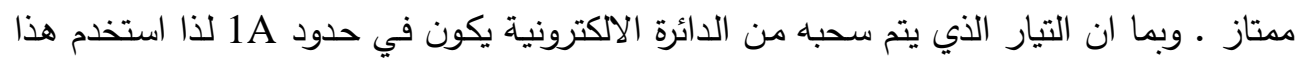
النوع من المنظمات الذي يجهز فولتية قدرها 5V+ وبتيار اخراج قدره 1.5A او اكثر بقليل . 
في عملية برمجة او مسح الذاكرة الومضية التي تحتاج الى فولتية برمجة عند الطرف

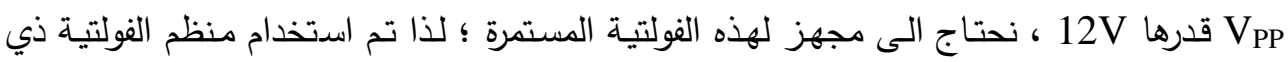
الرقم (7812) الذي يقوم بتحويل الفولتية المستمرة التي تدخل اليه الى فولتية مستمرة منظمة قدرها

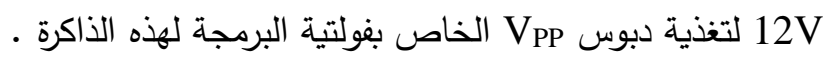

\section{* منظم الفولتية 7815}

عندما يراد برمجة او مسح الذاكرة الومضية التي تحتاج الى هذا الدبوس يجب ان يجهز

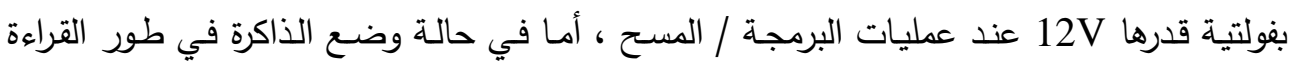

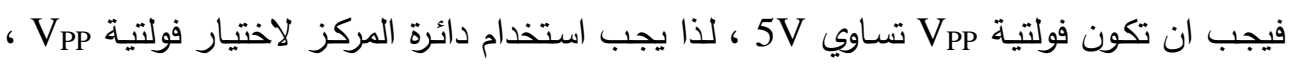

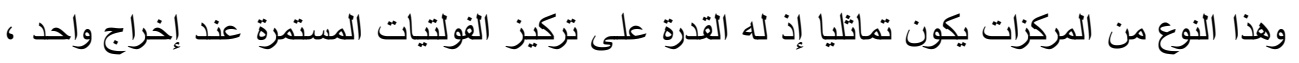

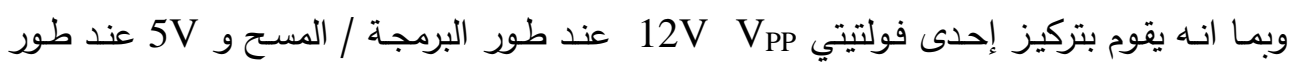

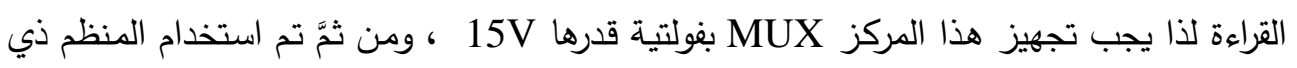

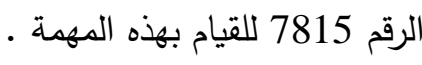

\section{FM منظم الفولتية L 317}

لغرض تطوير جهاز المبرمج برمجة ذاكرات من نوع PROM أو EPROM فأن فولتية

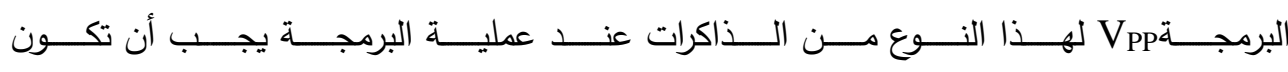
12.7 12.9V

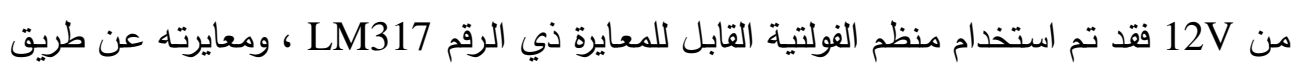

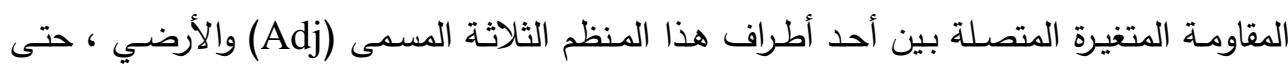
تساوي فولتية إخراجه 12.8V+ تقريبا وهي الفوليتة اللازمة لعملية برمجة PROM و

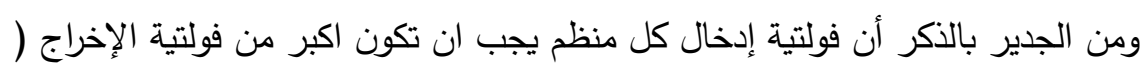

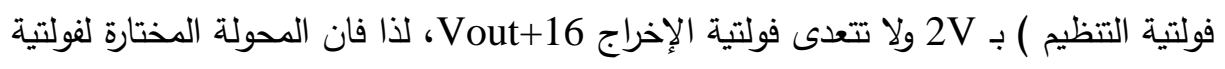
إخراج 18V تفي لهذا الغرض. 


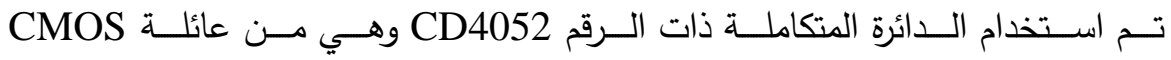
والتي تمثل دائرة اختيار لأربع (Complementary of Metal Oxide Semiconductor) قنوات تماثلية لكي تتصل إحداها بإخراج الدائرة ـ وقد تم اختيار إحدى دوائر الاختيار الأربع قنوات

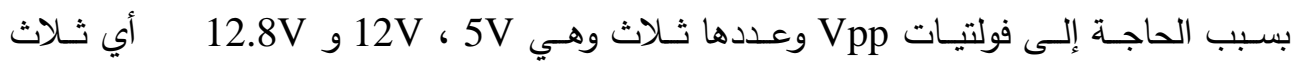
فولتيات.أما الجزء الثاني من الدائرة المتكاملة فهو غير مستخدم لعدم الحاجة اليه .

4-2-2 سجلات العنوان

تم استخدام ثلاث دوائر متكاملة ذوات الرقم 74LS374 إذ أن كل واحدة منها تحتوي على ثماني مراجيح من نوع D-type Flip Flop والتي تستخدم لتجهيز خطوط العنوان الخاصة بالذاكرة المراد القراءة منها او الكتابة فيها او مسحها بالعنوان المطلوب ، والكاب والسبب في استخدام ثلاث

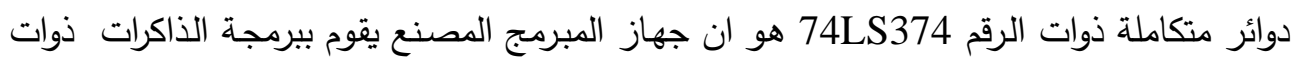

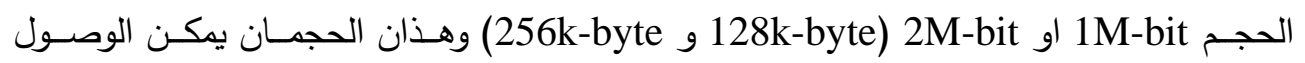

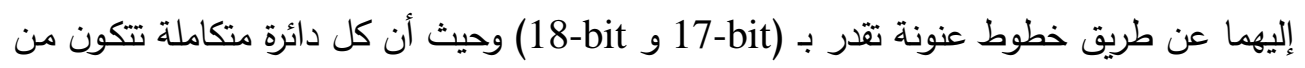
8-Bit Register لذا استخدمنا ثلاث منها ـ كما ويمكن تطوير الجهاز لكي يقوم ببرمجة أحجام اكبر بسبب وجود عدد من الـ Bits غير مستخدمة (Free ) في السجل الثالث.

5-2-2

يستخدم هذا السجل لتجهيز الذاكرة المراد برمجتها أو مسحها بالبيانات المطلوبة ، حيث تم استخدام الدائرة المتكاملة 74LS374 أيضا لهذا الغرض ، نخزن البيانات المرسلة من منفذ الطابعة

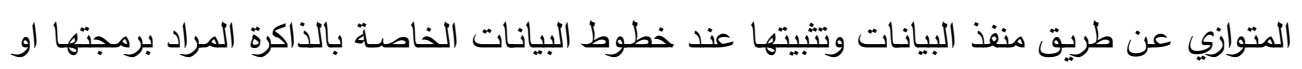

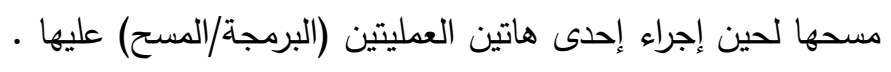

6-2-2 سجل إدخال البيانات

لغرض قراءة البيانات من الذاكرة المراد القراءة منها ، تم استخدام سجل إدخال والمتمثل بالدائرة المتكاملـة ذات الرقم تستخدم عادة بوصفها سجل إدخال في اكثر التطبيقات ، كما أن هناك فائدة كبيرة لهذا السجل في هذا التصميم تتمثل بمنع حدوث النزاع على خطوط البيانات بين الخطوط الخاصـة بالذاكرة المراد برمجتها وخطوط البيانات الخاصة بمنفذ الطابعة المتوازي. 
7-2-2

تم استخدام الدائرة المتكاملة ذات الرقم 74LS374 كذلك بوصفها سجل إخراج من مهامه

إخراج قيم إشارات السيطرة المتمثلة بـ CE\#, OE\#, WE\# للذاكرة المراد برمجتها او القراءة منها ،

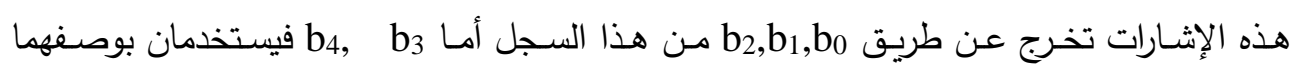

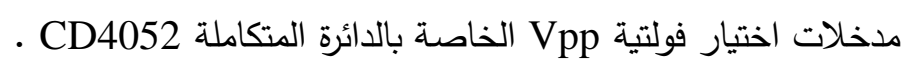

\section{8-2-2}

بما أن أحد محاور تصميم جهاز المبرمج هو ربط طريق منفذ الطابعة المتوازي عبر كابل بيانات لمسافة بعيدة نسبيا ، فقد تم استخدام الدائرة المتكاملة ذات الرقم

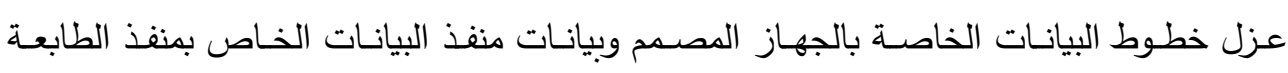

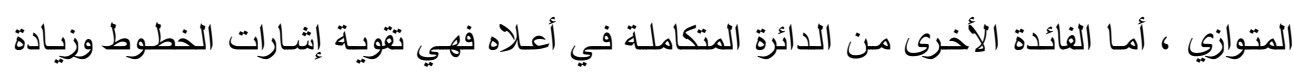
لمنع حدوث اي خطأ في أثناء عملية النقل بين منفذ الطابعة المتوازي وجهاز المبرمج. Fan-Out

9-2-2

تم استخدام الدائرة المتكاملة ذات الرقم 74LS244 لعزل خطوط السيطرة الخاصـة بمنفذ

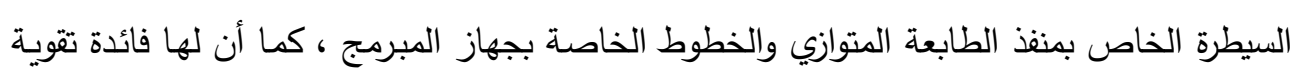
الإشارات الخاصة بالسيطرة وزيادة Fan-Out .

10-2-2

تم استخدام الدائرة المتكاملة المعروفة والمشهورة ذات الرقم 74LS138 التي تمثل 8-3

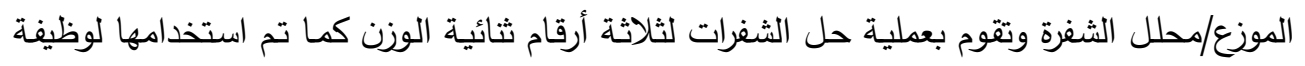
التوزيع للسيطرة على عملية تمكين إشارات السيطرة الخاصة بجميع سجلات الإدخال والإخراج فضلا

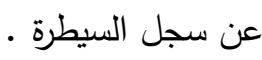

\section{3-2 عملية القراءة من الأكرة الومضية}

تتطلب عملية القراءة من الذاكرة عدة مراحل إذ يجب أولا إرسال عنوان الموقع المراد القراءة

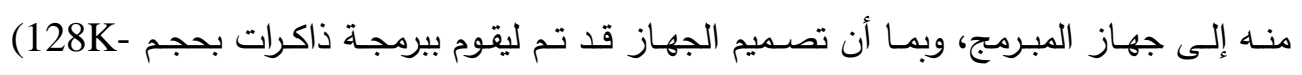




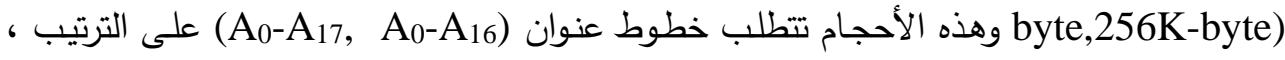

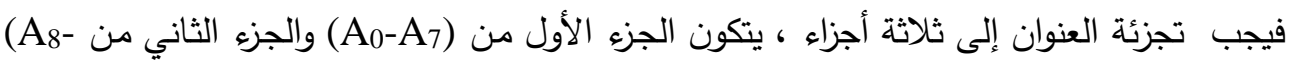

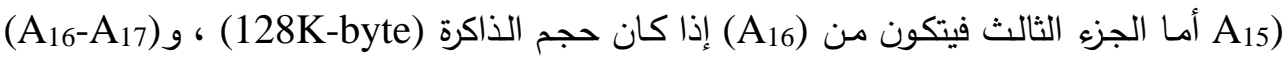
إذا كان حجم الذاكرة (256K-byte) • وقد تمت تجزئة العنوان إلى هذه الأجزاء بسبب أن منفذ إنذ البيانات الخاص بمنفذ الطابعة المتوازي الذي سوف يتم إرسال أجزاء العنوان من خلالهه إلى جهاز المبرمج يكون بعرض ثمانية أرقام ثنائية .

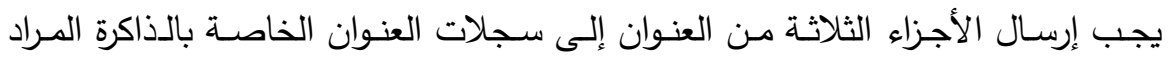

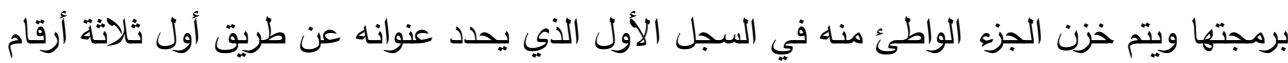

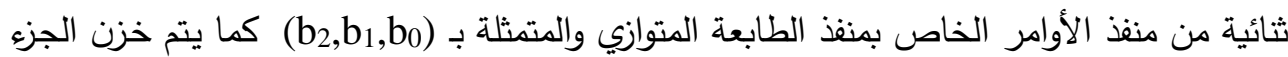
الوسطي في السجل الثاني ، أمسا الجزء العـالي فيخزن في السجل الثالث. والجدول (1) يوضـح عناوين كل من السجلات الخاصة بجهاز المبرمج • بعد إتمام عملية إرسال العنوان يتم تحديد مسار البيانات لمنفذ البيانات الخاص بمنفذ الطابعة المتوازي عن طريق تثبيت قيمة (b5=1 ) في سجل الأوامر ، ثم يتم إرسال إشارة السيطرة عن طريق سجل الأوامر الخاص بالجهاز المصمح لكي تطبق على الثريحة المراد القراءة منها ثم ترسل إشارة OE\# إلى الثريحة نفسها لتمكين خطوط البيانات الخاصة بالذاكرة من إخراج

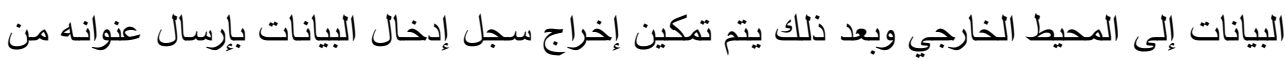
خلال محلل الثفرة. تنساب البيانات الخارجة بعد ذلك في خطوط البيانات الخاصـة بجهاز المبرمج ومن الجدير بالذكر أن من الضروري بعد جميع العمليات أعلاه أن يتم إرجاع قيم إثارات السيطرة

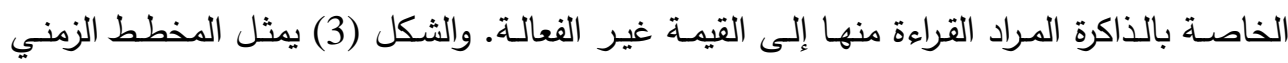

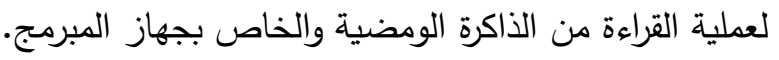

الجدول (1): عناوين السجلات الخاصة بجهاز المبرمج

\begin{tabular}{|c|c|c|c|}
\hline \multirow[b]{2}{*}{ السجل } & \multicolumn{3}{|c|}{ سجل الأوامر لمنفذ الطابعة المتوازي } \\
\hline & $\begin{array}{c}\text { CMD2 } \\
\mathrm{B}_{2} \\
\end{array}$ & $\begin{array}{c}\text { CMD1 } \\
\text { B }_{1}\end{array}$ & $\begin{array}{c}\text { CMD0 } \\
\text { B }_{0} \\
\end{array}$ \\
\hline
\end{tabular}




\begin{tabular}{|c|c|c|c|}
\hline سجل جزء العنوان الواطئ (A0-A7) & $\mathbf{0}$ & $\mathbf{0}$ & $\mathbf{0}$ \\
\hline سجل جزء العنوان الوسطي (A8-A15) & $\mathbf{0}$ & $\mathbf{0}$ & 1 \\
\hline سجل جزء العنوان الوسطي (A16 : A10) ، (A17:020) & $\mathbf{0}$ & 1 & $\mathbf{0}$ \\
\hline سجل الأوامر & $\mathbf{0}$ & 1 & 1 \\
\hline سجل إخراج البيانات & 1 & $\mathbf{0}$ & $\mathbf{0}$ \\
\hline سجل إدخال البيانات & 1 & $\mathbf{0}$ & 1 \\
\hline غير مستخدم & 1 & 1 & $\mathbf{0}$ \\
\hline 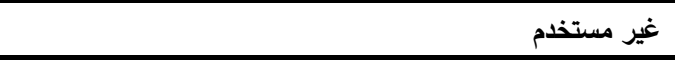 & 1 & 1 & 1 \\
\hline
\end{tabular}

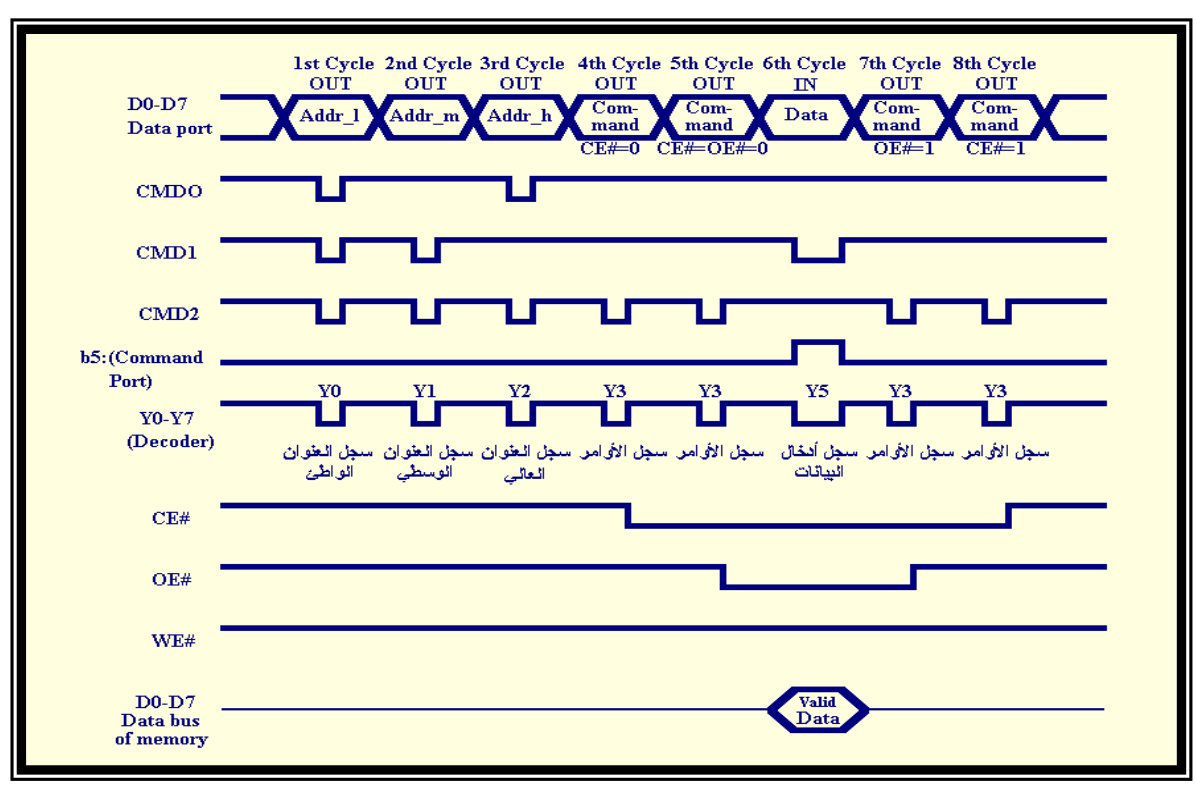

الشكل (3): المخطط الزمنى لعملية القراءة من الذاكرة الومضية الخاص بجهاز المبرمج

4-2 عملية الكتابة في الذاكرة الومضية : الكطط الزمنية

يتم إرسال العنوان الخاص بالموقع المراد الكتابة فيه بالطريقة نفسها تمامـا والمتبعة في عملية القراءة من الذاكرة ، بعد ذلك يتم تحديد فولتية البرمجة (Vpp) المناسبة للذاكرة المراد الكتابة

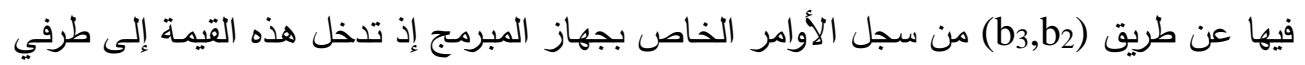

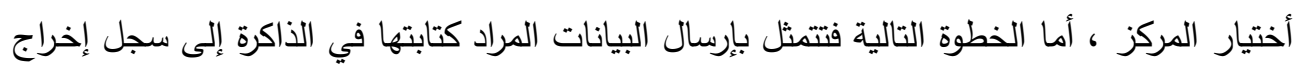
البيانات بعد جعل قيمة (b5=0) في منفذ الأوامر الخاص بمنفذ الطابعة المتوازي لكي يكون الجاه لئاه

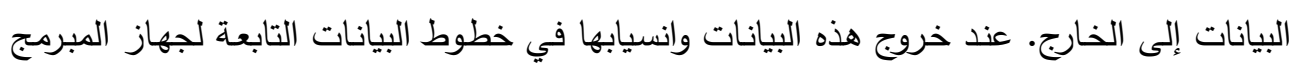

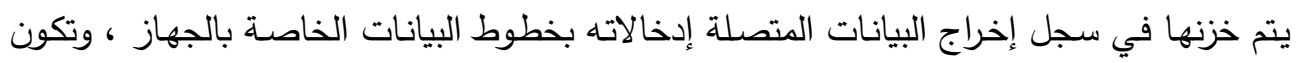


إخراجـات هذا السـل متصـلة بخطوط البيانـات الخاصـة بالذاكرة المـراد كتابتها فيهـا ، يلي هذه

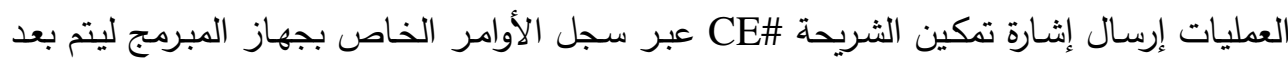
ذلك إرسال إثارة تمكين الكتابة WE\# عبر السجل نفسه لكي تتم الكتابة في الذاكرة .

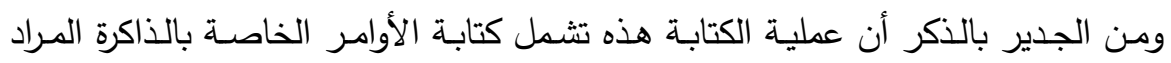
برمجتها أو البيانات التي تتم برمجتها في الذاكرة ـ وقد تم إرجاع جميع قيم إثارات السيطرة الخاصة

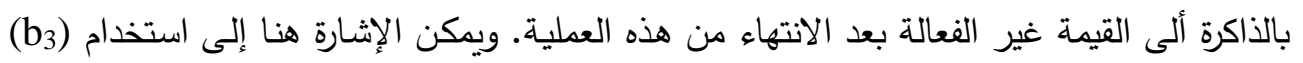

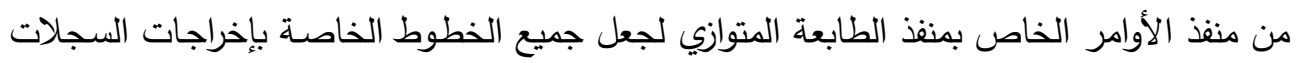
الخاصة بجهاز المبرمج والمتصلة بالذاكرة المراد برمجتها ومن ضمنها طرفي التغذية فعالة أو خاملة

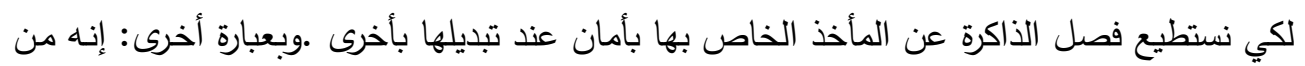

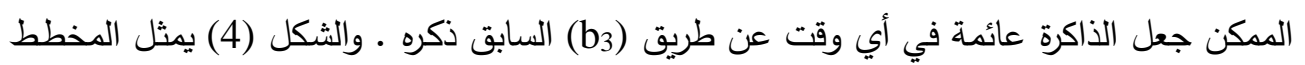

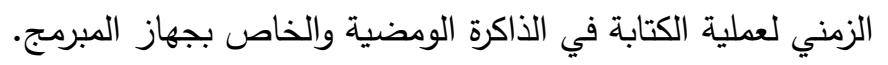

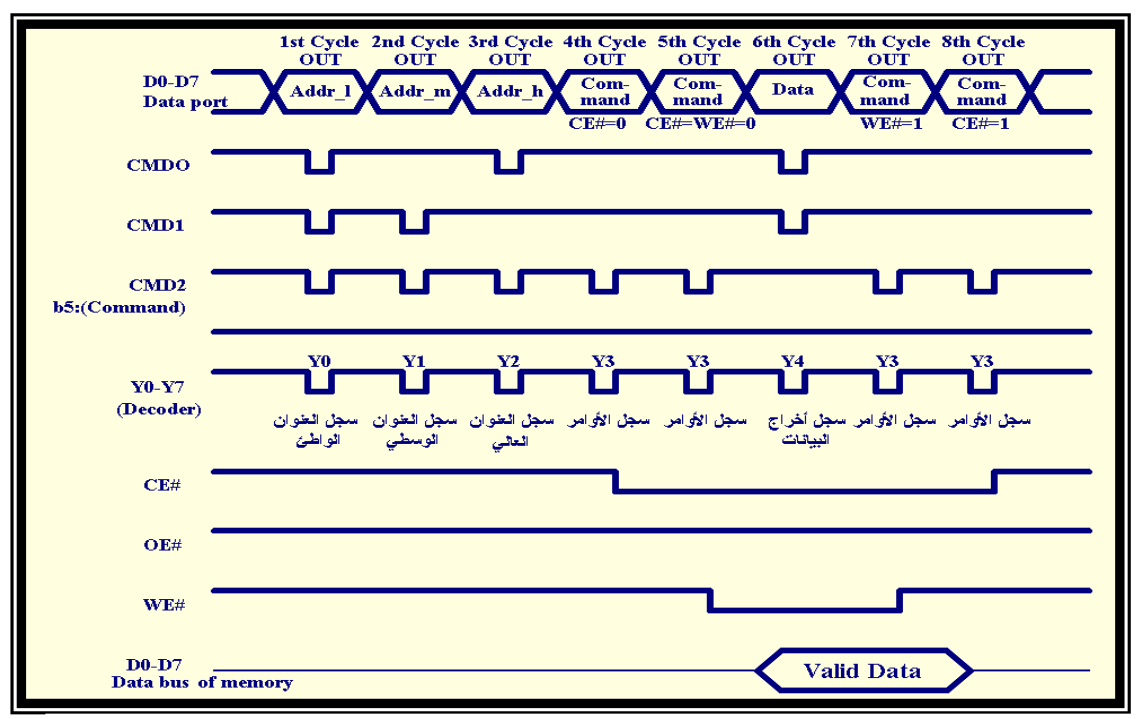

الشكل (4): المخطط الزمنه، لعملية الكتابة فه، الذاكرة الومضية وإلخاص بجهاز.

3- الوصف البرمجي لجهاز المبرمج

تم استخدام لغة (C++) لبناء البرنامج والدوال الخاصـة بـه لما تتمتع بـه هذه اللغـة من

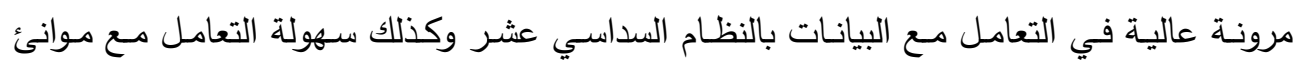


الإدخال والإخراج وباستخدام دوال مكتبية بسيطة وغير معقدة فضلا عن توافر إمكانات عالية في حل أغلب المشاكل التي قد تظهر في اللغات الأخرى. 1-3 الهيكل العام للبرنامج

أن الهيكل العام للبرنامج يتكون من عدة أجزاء وكما موضح في الثكل (5).

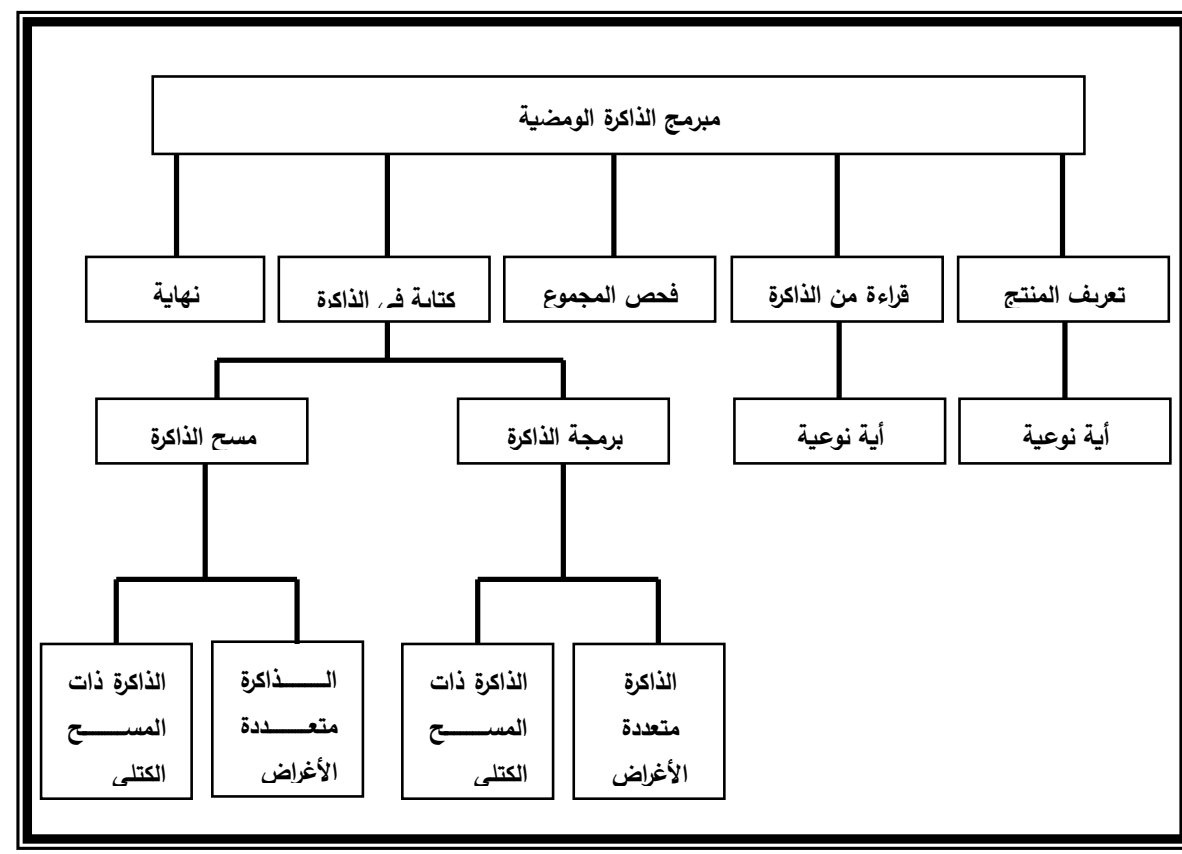

الشكل (5) المخطط العام لبرنامج مبرمج الذاكرة الومضية

Product ID 1-1-3 تعريف المنتج

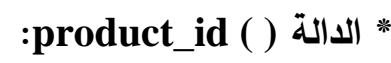

تستخدم هذه الدالة للكثف عن الذاكرة الومضية متعددة الاغراض MPF والتي تحمل الرقم

1.استدعاء الدالة Write-byte لكتابة القيمة (AAH) في الموقع (5555H). 2.استدعاء الدالة Write-byte لكتابة القيمة (55H) في الموقع (2AAAH). 3. استدعاء الدالة Write-byte لكتابة القيمة (90H) في الموقع (5555H). 
4.استدعاء الدالة read-byte لقراءة البايت الأول الذي يمثل رمز الثركة المصنعة من العنوان

.$(0000 \mathrm{H})$

5.استدعاء الدالة read-byte لقراءة البايت الاول الذي يمثل رمز الثركة المصنعة من العنوان

.$(0001 \mathrm{H})$

6.استدعاء الدالة write-byte لكتابة القيمة (AAH) في الموقع (5555H).

7. استدعاء الدالة write-byte لكتابة القيمة (55H) في الموقع (2AAAH) في الدوقع (2AH).

8. استدعاء الدالة write-byte لكتابة القيمة (FOH) في الموقع (5555H).

ملاحظة: * الخطوات 1 , 2, 3 تستخدم للاخول إلى طور تعريف المنتج •

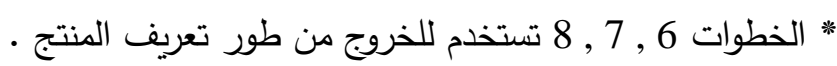

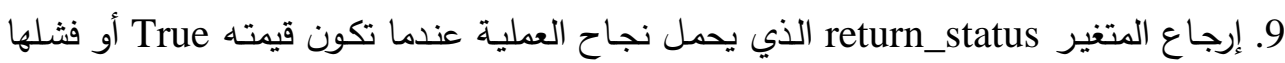

عندما تكون قيمته false

\section{: write-byte (a-h, a-m, a-l, dat) الدالة * *}

تستخدم هذه الدالة لكتابة بايت واحد في العنوان المحدد بأجزاء العنوان الثلاثة المدخلة

إلى هذه الدالة .

الخوانمية :

1. send-address ( ) (استدعاء الدالة

2.write-data ( ) (استدعاء الدالة

\section{:send_address (addr_h,addr_m,addr-l) الدالة *}

تستخدم هذه الدالة لإرسال العناوين الخاصة بالذاكرة المراد القراءة منها أو الكتابة فيها.يتم

إرسال هذه العناوين على ثلاث مراحل ، يتم في كل مرحلة منها خزن جزء من العنوان في أحد الإدسال

$$
\text { سجلات العنوان للجهاز المبرمج • الفولى }
$$

الخوارنمية : الخدوان

1. إرسال القيمة (08H) إلى منفذ الأوامر ، لإلغاء تفعيل جميع إخراجات محلل الثفرة الخاص بعملية الكتابة في السجلات الخاصة بجهاز المبرمج.

2. إرسال جزء من العنوان الواطئ (addr-1) الى منفذ البيانات لكي يتم خزنه في السجل الخاص لئه 
3. إرسال القيمة (0FH) الى منفذ الأوامر لتفعيل اشارة strobe الخاصة بالسجل الاول .

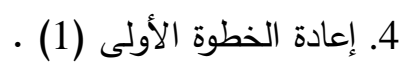
5. إرسال جزء العنوان الوسطي (addr-m) الى منفذ البيانات لكي يتم خزنه في السجل الخاص به 6.إرسال القيمة (0EH) الى منفذ الأوامر لتفعيل اشارة strobe الخاص بالسجل الثاني .

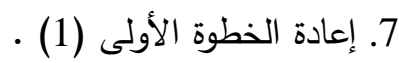
8. إرسال جزء من العنوان العالي (addr-h) الى منفذ البيانات لكي يتم خزنه في سجله الخاص. 9. إرسال القيمة (0DH) الى منفذ الأوامرلتفعيل اشارة

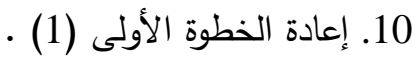

\section{:write-data (dat) الدالة *}

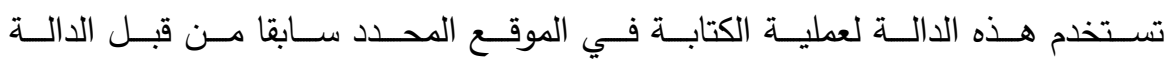

.send_address

\section{الخوارنمية :}

1.ارسال القيمة (08H) إلى منفذ الأوامر لتأكيد إلغاء تفعيل إخراجات محلل الثفرة .

2. إرسال البيانات (dat) إلى منفذ البيانات المراد كتابتها في الذاكرة .

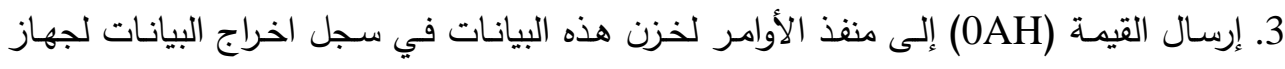

- المبرمج

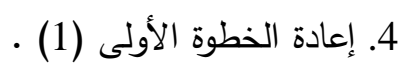

5. إرسال القيمة (OFH) إلى منفذ البيانات لكي تخزن في سجل الأوامر الخاص بجهاز المبرمج ،

جعل قيمة CE\# و OE\# و WE\# عند الحالة المنطقية العالية .

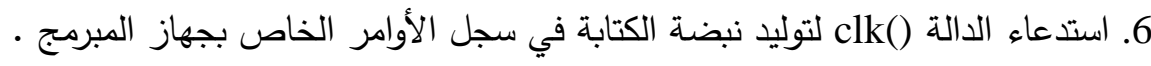

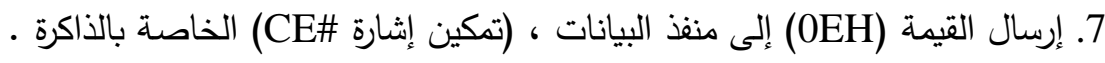

8. إعادة الخطوة السادسة .

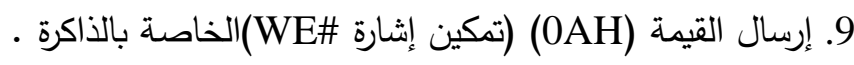

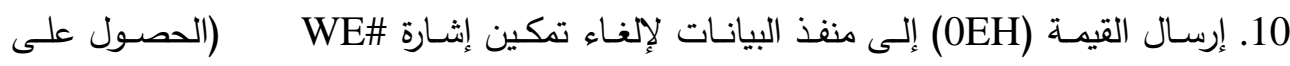

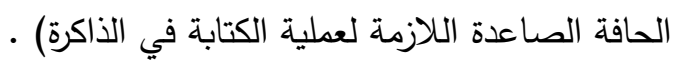

11. إعادة الخطوة السادسة (6) . 
12. إرسال القيمة (0FH) إلى منفذ البيانات لإلغاء تمكين إثارة CE\# .

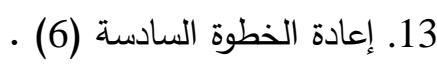

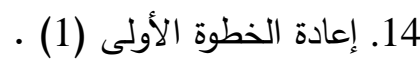

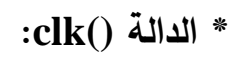

تستخدم هذه الدالة لإرسال نبضة للحصول على حافة صاعدة يحتاج اليها سجل الاوامر

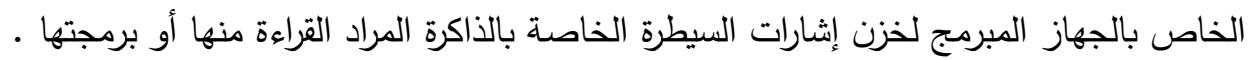

الخوارنمية :الخاصن

1. إرسال القيمة (08H) إلى منفذ الأوامر (الحصول القيمة المنطقية العالية). 2. إرسال القيمة (OCH) إلى منفذ الأوامر (للحصول على القيمة المنطقية الواطئة). 3. إرسال القيمة (08H) مرة أخرى إلى منفذ الأوامر (للحصول على الحافة الصاعدة).

2-1-3 القراءة من الذاكرة الومضية Read FLASH:

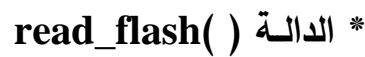

تستخدم هذه الدالة لقراءة محتويات اي نوع من انواع الذاكرات وخزن البيانات المقروءة في ملف محدد . م م

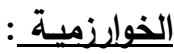
1. قراءة اسم الملف المراد قراءة البيانات من الذاكرة وخزنها فيه ـ.

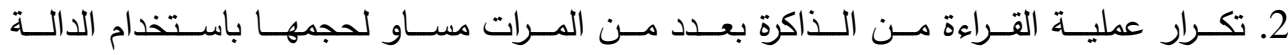
.read-byte

:f-name (f-n[10]) الدالة *

تستخدم هذه الدالة لتحديد وقراءة اسم الملف الذي سوف تتم القراءة منهه لغرض خزن محتوياته في الذاكرة المراد برمجتها أو كتابة البيانات في الملف نفسه التي تتم قراءتها من الذاكرة. الخوانمية : 
1. قراءة اسم الملف من لوحة المفاتيح ، يتكون اسم الملف من 11 حرفاً على الأكثر، وثمانية أحرف لاسم الملف وثلاثة أحرف للامتداد. 2. إرجاع اسم الملف إلى الدالة التي قامت باستدعاء هذه الدالة.

: read-byte (a-b, a-m, a-l) الدالة *

تستخدم هذه الدالة لقراءة بايت واحد من العنوان المحدد بأجزاء العنوان الثلاثة المدخلة إلى

هذه الدالة .

الخوارزمية:

1 . send-address( ) استدعاء الدالة

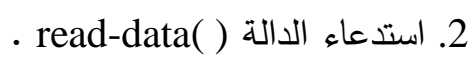

3. إرجاع قيمة dat إلى الدالة المستدعية .

\section{:read-data ( ) الدالة * *}

تستخدم هذه الدالة لعملية القراءة من أي نوع من أنواع الذاكرات التي يراد القراءة منها، ذلك

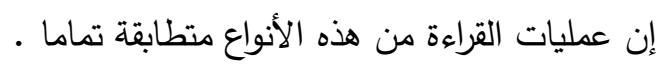

الخوارنية :

1. إرسال القيمة (08H) إلى منفذ الأوامر لتأكيد إلغاء تمكين إخراجات محلل الثفرة.

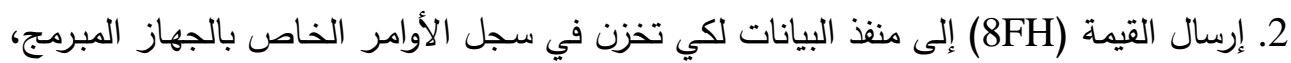

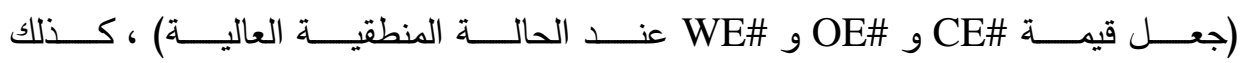
جعل by =1 من هذا السجل لتحديد اتجاه البيانات ، (جعل إخراجات سجل إخراج البيانات الخاص بالجهاز المبرمج سائبا).

3. استدعاء الدالة clk لتوليد نبضة الكتابة في سجل الأوامر الخاص بالجهاز المبرمج .

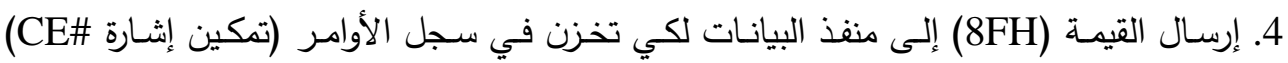
الخاص بالذاكرة .

5. إعادة الخطوة الثالثة (3) ـ الثرة.

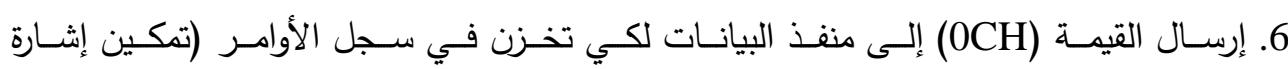

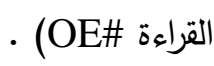

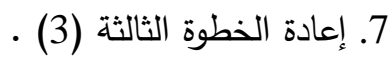


8. إرسال القيمة (28H) إلى منفذ الأوامر لمنفذ الطابعة (إلغاء تمكين إخراجات الثفرات) مع تحديد

اتجاه البيانات الخاص بمنفذ البيانات (جعل حركة البيانات داخلة) .

9. إرسـال القيمة (2BH) إلى منفذ الأوامر لكي يتم تمكين إخراج سجل إدخال البيانات الخاص

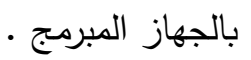

10. بعد انسياب البيانـات في خطوط البيانات الخاصـة بالجهاز المبرمج تتم قراءتها عبر منفذ

البيانات باستخدام الأمر

11. إرسال القيمة (28H) الى منفذ الأوامر لالغاء تمكين اخراجات محلل الثفرة.

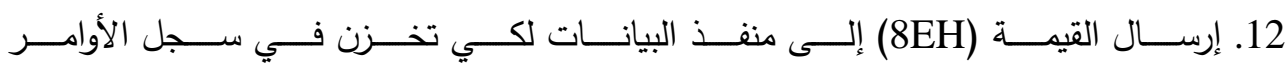

• (جE\#=1 (جعل قيمة)

13. إعادة الخطوة الثالثة (3).

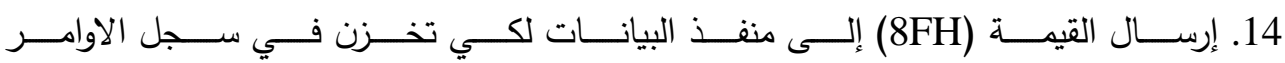

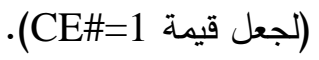

15. إعادة الخطوة الثالثة (3).

16. إرسال القيمة (0FH) إلى منفذ البيانات لتمكين مخرجات سجل اخراج البيانات.

17. إعادة لخطوة الثالثة (3).

18. إرسال القيمة (08H) إلى منفذ الأوامر لإلغاء تمكين إخراجات محلل الشفرة وكذلك إعادة اتجاه

حركة البيانات من خلال منفذ البيانات.

1-2-3 الكتابة في الذاكرة الومضية Write FLASH:

فيتم استدعاء الدالة .MPF: 39SF02 فئل

الذاكرة بالكامل .أمسا إذا كان نوع الذاكرة ذات المستح الكتلي BE:28F020 فيتم استدعاء الدالة

.BE-program(er)

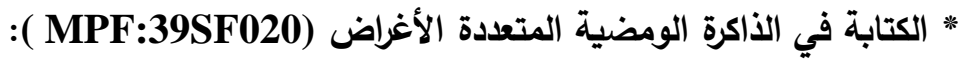

تتم الكتابة في هذا النوع من الذاكرات باستخدام الدالة () MPF-Program التي تقوم

بيرمجة الثريحة ، (Sector) بعد الآخر . بلهن

MPF-Program() الدالة * * 
تستخدم هذه الدالـة لبرمجة الذاكرة الومضية متعددة الأغراض بأكملها (Sector) بعد

\section{الخوارزمية:}

1. استدعاء الدالة f- name لقراءة اسم الملف المراد القراءة منه من لوحة المفاتيح •

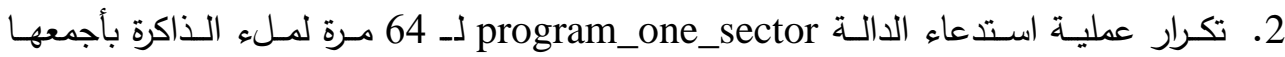

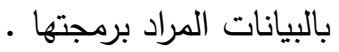

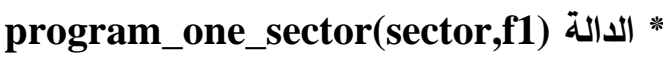

تستخدم هذه الدالة لبرمجة كتلة واحدة محددة بالعنوان (sector) المدخل الى هذه الدالة.

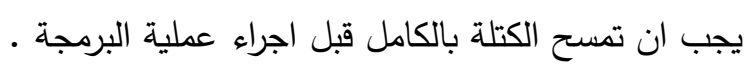

الخوارزمية:

1. استدعاء الدالة erase_one_sector لمسح الكتلة ابتداء بالعنوان (sector) . 2. تكرار استدعاء الدالة program_one_byte لـ (4096) مرة لملء الذاكرة بالبيانات المراد

:erase-one-sector(sect) الدالة * * تستخدم هذه الدالة لمسح كتلة متكونة من 4k-byte من الذاكرة ابتداء بالموقع sect ـ

الخوارزمية:

1. استدعاء الدالة write-byte لكتابة الامر (AAH) في الموقع (5555H) .

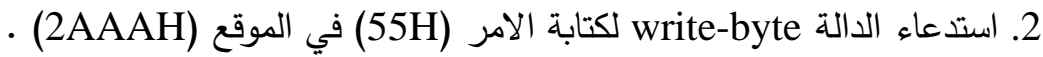

3. استدعاء الدالة write-byte لكتابة الامر (80H) في الموقع (5555H) .

4. استدعاء الدالة write-byte لكتابة الامر (5AH) في الموقع (555H) .

5. استدعاء الدالة write-byte لكتابة الامر (55H) في الموقع (2AAAH) .

6. استدعاء الدالة write-byte لكتابة الامر (30H) في الموقع (sect) •

7. استدعاء الدالة c-t-r للكثف عن اكتمال عملية مسح المقطع الداخلية للشريحة .

: program-one-byte(adh,adm,adl,dt) الدالة * * 
تستخدم هذه الدالة لبرمجة بايت واحد (dt) في العنوان المحدد بـاجزاء العنوان الثلاثة

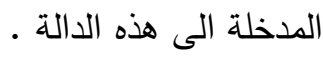

الخوانزمية:

1. استدعاء الدالة write-byte لكتابة الأمر (AAH) في الموقع ( 555H).

2. استدعاء الدالة write-byte لكتابة الأمر (55H) في الموقع ( 1 (2AAAH).

3. استدعاء الدالة write-byte لكتابة الأمر (A0H) في الموقع ( 1 (555H).

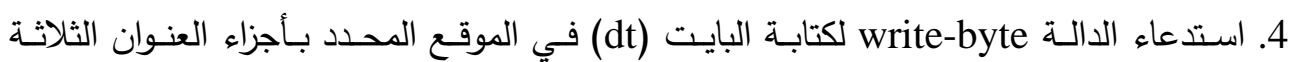

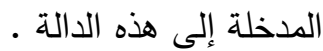

5. استدعاء الدالة c-t-r للكثف عن اكتمال عملية البرمجة الداخلية للبايت.

:c-t-r (a-h, a-m, a-l) الدالة *

وظيفة هذه الدالة هي نفسها في الدالة السابقة .

الخوانمية:

1. استدعاء الدالة (read-byte(لقراءة البايت الذي تمت كتابته لاحقا من العنوان نفسه الذي كتبت

فيه .

2. حجب جميع الـ bits الخاص بالبايت عدا bit-6 .

3. تكرار عملية القراءة من الموقع نفسه الى ان نحصل على الـ bit نفسه في المرة الاولى . عند

ذاك تكون عملية الكتابة قد اكتملت داخليا .

• الكتابة في الذاكرة الومضية ذات المسح الكتلي (BE:28F020):

تتم الكتابة في هذا النوع من الذاكرات باستخدام الدالة (BE-program التي تقوم بيرمجة

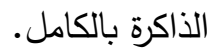

:BE-Program(er) الدالة * *

تستخدم هذه الدالة لبرمجة الذاكرة الومضية ذات المسح الكلي . اعتمد في هذه الدالة على

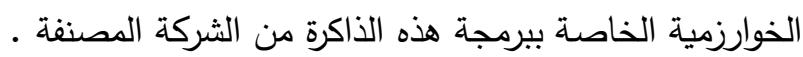


الخوازيمية:

1. استدعاء الدالة f-name لقراءة اسم الملف المراد القراءة منه في لوحة المفاتيح .

2. تهيئة العنوان (address=0) وتهيئة فولتية البرمجة (Vpp=12V) .

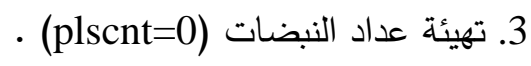

4. قراءة byte من الملف المحدد في الخطوة (1) .

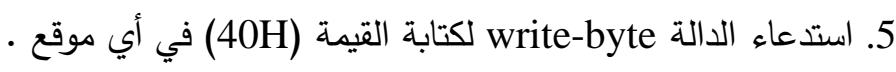

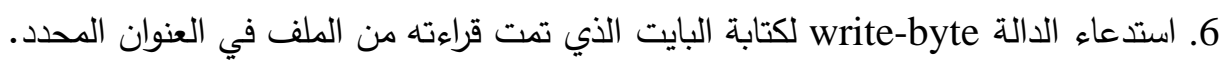

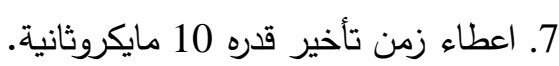

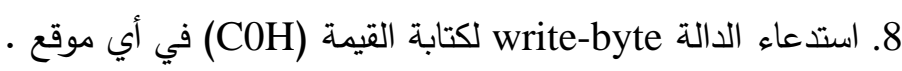

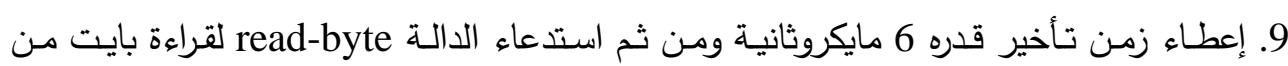

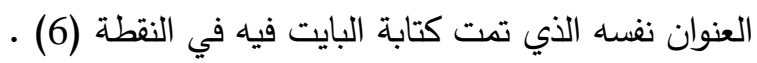

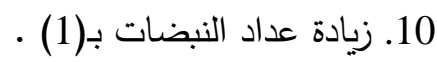

11. عند عدم مطابقة البايت الذي تمت برمجته في الخطوة (6) للبايت الذي تمت قراءتها في

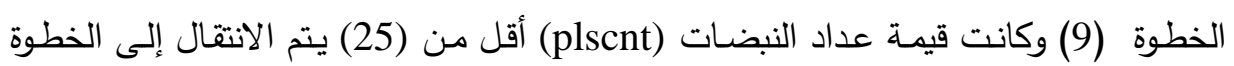

12. إذا كان العنوان اقل من 40000H اعد الخطوات السابقة ابتداء من الخطوة (3) .

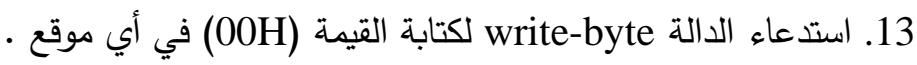

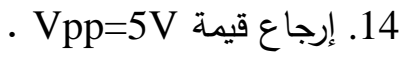

15. يتم إرجاع القيمة (1) عند نجاح عملية البرمجة أو القيمة (1) عند فثلها .

4-1-3 مسح الذاكرة الومضية Erase-FLASH:

إذا كان نوع الذاكرة MPF:39F020 فيتم استدعاء الدالة (2) erase-entitre-chip( ـ أما

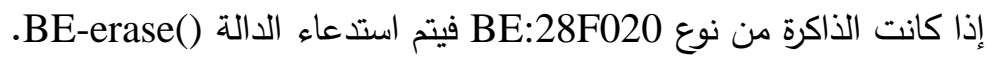

• مسح الذاكرة الومضية المتعدة الأغراض (MPF:39SF020)

: erase-entire-chip() الدالة * 


\section{الخوارزمية:}

تستخدم هذه الدالة لمسح محتويات مصفوفة الذاكرة بأكملها .

1. استدعاء الدالة write-byte لكتابة القيمة (5AH) في العنوان (555H).

2. استدعاء الدالة write-byte لكتابة القيمة (55H) في العنوان (2AAAH).

3. استدعاء الدالة write-byte لكتابة القيمة (80H) في العنوان (5555H).

4. استدعاء الدالة write-byte لكتابة القيمة (AAH) في العنوان (5555H).

5. استدعاء الدالة write-byte لكتابة القيمة (55H) في العنوان (2AAAH).

6. استدعاء الدالة write-byte لكتابة القيمة (10H) في العنوان (5555H)

7. استدعاء الدالة d-p أو c-t-r للكثف عن اكتمال عملية المسح الداخلي للشريحة بأكملها.

(BE:28F020) مستح الذاكرة الومضية ذات المسح الكتلي

:BE-erase() (الدالة :

الخوارزمية:

1. تهيئة فولتية البرمجة (Vpp=12V) .

2. استـعاء الدالة be-program لخزن القيمـة (0) في جميـع المواقع الخاصـة بالذاكرة واستلام

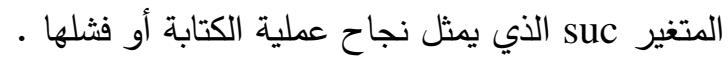

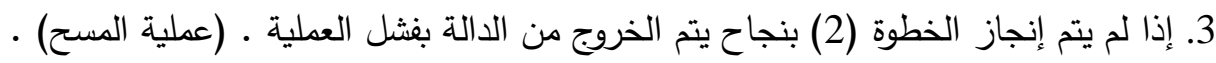

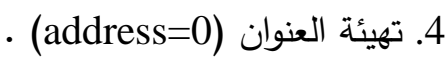

5. تهيئة عداد النبضـات (plscnt=0) • والمتغير (stat=0) الذي يمثل حالـة التسلسل لعمليات

الكتابة .

6. إذا كانت قيمة (stat=1) فيتم الانتقال إلى الخطوة (10) • (لكتئ

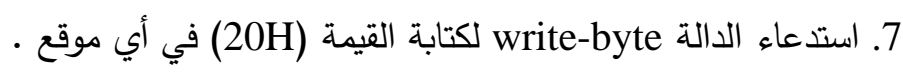

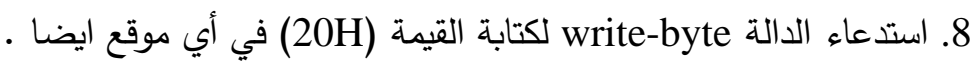

9. إعطاء زمن تأخير قدره 10 ms.

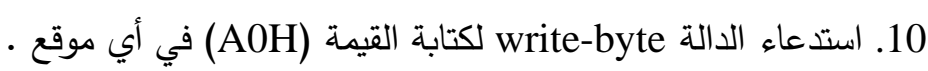

11. إعطاء زمن تأخير قدره $6 \mu$ second

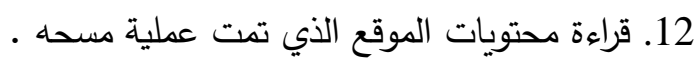


13. مقارنة البايت المقروء في الخطوة (12) بالقيهة (FFH). يتم الانتقال إلى الخطوة (7) عند

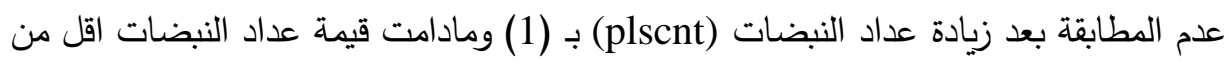
3000 .وإلا يتم الخروج من الدالة بفثل العملية ـ (عملية المسح) .

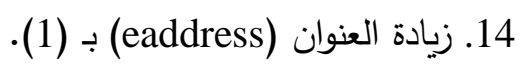

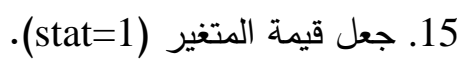

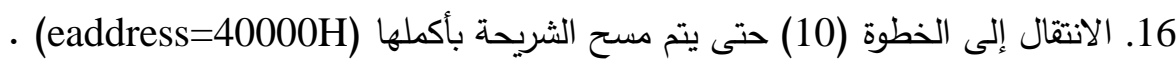

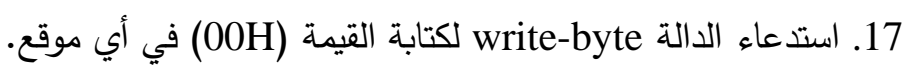

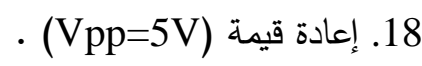
19. يتم إرجاع القيمة (1) عند نجاح عملية الدسح او القيمة (1) عند فثلها .

\section{5-1-3 فصص المجموع CHECHSUM:} :check_sum (c-s) الدالـة *

تستخدم هذه الدالة لجمع محتويات الثريحة بأكملها لاكتثاف أية حالة خطأ في عملية نقل

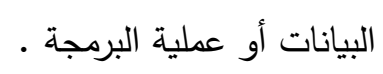

\section{الخوالنميـة:}

1. 1. طباعة ناتج جمع محتويات الذاكرة على شاشة العرض. 2. انتظار الضغط على أي مفتاح للرجوع الى الدالة المستدعية لهذه الدالة.

\section{:End 6-1-3}

يتم استدعاء الدالة (لفئل off( لفصل دبابيس الذاكرة عن جهاز المبرمج لرفعها من المقبس

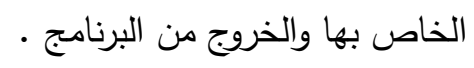

$$
\text { off() : الدالة * * (2) }
$$

تستخذم لفصل دبابيس الذاكرة كهربائيا من أي مصدر إشارة سواء أكانت إثارات منطقية

$$
\text { أم تغذية. }
$$

الخوارنمية:

1. إرسال القيمة (00H) إلى منفذ الأوامر . 
2. الانتظار إلى حين الضغط على أي مفتاح ضمن لوحة المفاتيح الخاصـة بالحاسوب لكي يتسنى للمستخدم رفع او تبديل الثريحة.

\section{4- الاستنتاجات}

تم تصميم جهاز مبرمج الذاكرة الومضية عن طريق وضع خارطة لربط الدوائر المتكاملة .

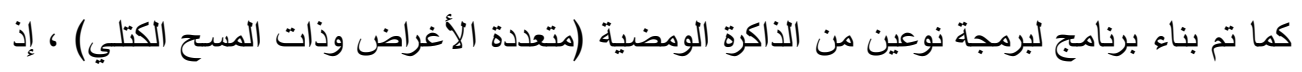
وفر البرنامج إمكان قراءة وبرمجة ومسح هذين النوعين من الذاكرة .

وتم تطبيق هذا النظام في عملية استتساخ وبرمجة هذين الصنفين من الذاكرة الومضية والتي تستخدم في اكثر اللوحات الرئيسة للحاسوب ـ وفيما يلي بعض من هذه الاستتناجات:

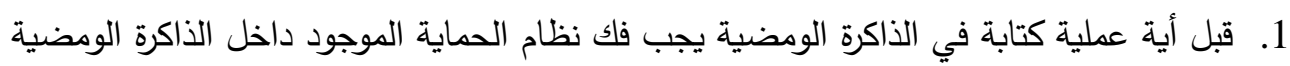

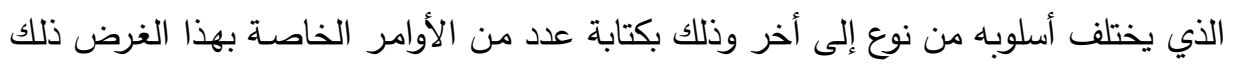

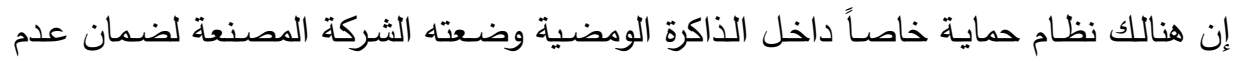
حدوث أية عملية كتابة عرضية في أثناء رفع او خفض القدرة عند إيقاف تثغيل الحاسوب ، ومن دون نظام الحماية المذكور سابقاً قد يتوقف الحاسوب عن العمل ولا يمكن إعادة تشغيله

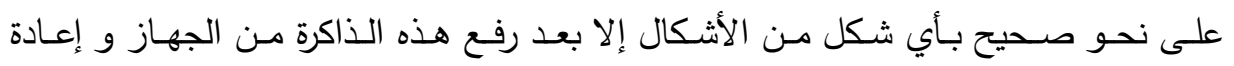

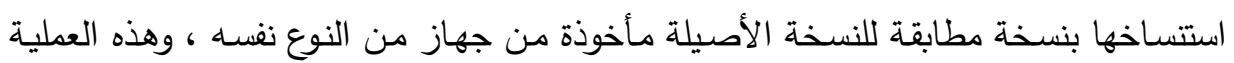

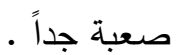
2. لا يمكن الكتابة في أي موقع ضمن مصفوفة الذاكرة الومضية إلا بعد أن يتم مسح محتويات هذا الموقع او مسح المقطع الذي يضم هذا الموقع او مصفوفة الذاكرة بأكملها.

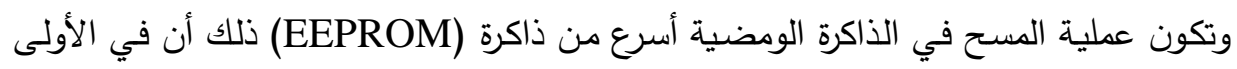
يمكن مسح (Block) كامل او الثريحة بأكملها في وقت واحد في حين لا يمكن في الثانية

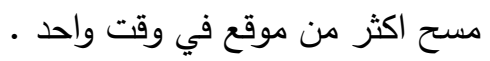

3. إن عددا من الفيروسات التي تتسبب في توقف الحاسبة عن العمل مثل فيروس (جيرنوبل) ليس

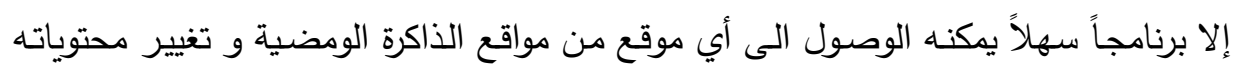
بمجرد فك الحماية عن عملية الكتابة في الذاكرة الومضية المخزون فيها النظام (BIOS) بعد موند معرفة عنوان بداية الذاكرة ضمن خارطة الذاكرة (Memory Map) .

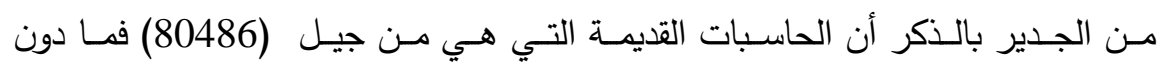

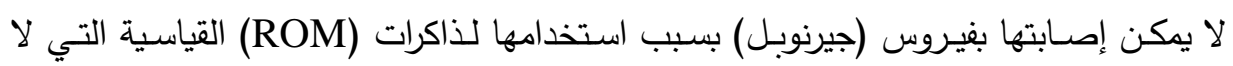


يمكن الكتابة فيها وهي متصلة بالجهاز المضيف إذ أنها تحتاج إلى فولتيات عالية في أثناء

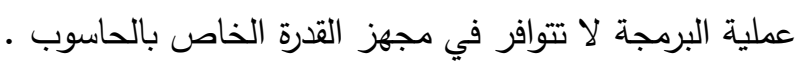

4. إن الـ(Flash Disk) هو نوع من أنواع الذاكرة الومضية التي لا تحتاج إلى فولتية برمجة عالية لإلية

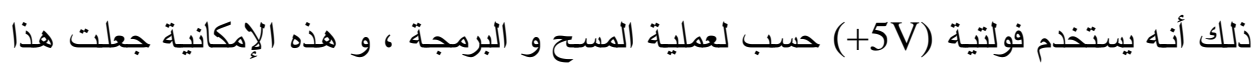

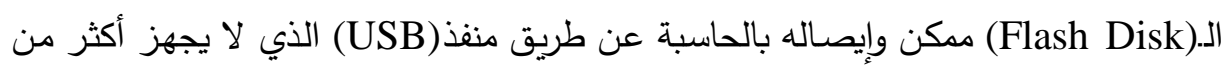
(5V)

5- التوصيات

نوصسي مستقبلا بتطوير جهاز المبرمج لغرض برمجـة أنواع مسن ذاكرات القراءة فقط أسط

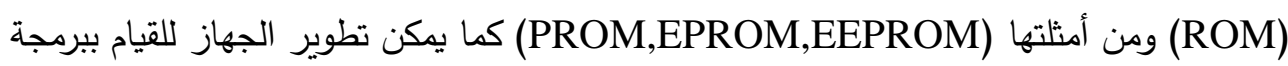
هذه الأنواع بأحجامها المختلفة ابتداء من اقل حجم إلى أكبر حجم. وكذلك يمكن تطويره لبرمجة نوع

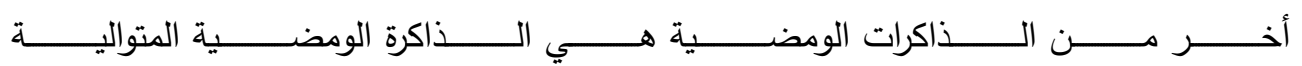
ولأن هذا التطوير يتطلب مكونات مادية إضافية كبيرة وتصميم دائرة معقدة للغاية (Serial Flash)

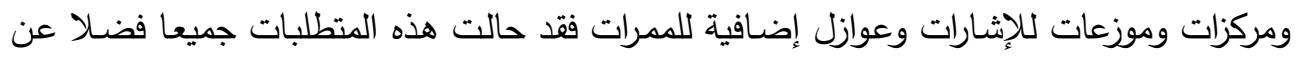
ضعف الإمكانات التقنية التي تحتاج إليها دون تطويره في الوقت الحاضر ل 


\section{المصادر}

[1] Advanced Micro Device, 1995, “Am28F020-2Megabit (262, 144×8Bit) CMOS 12.0 Volt, Bulk Erase Flash Memory", Data-Net 2.0.

[2] Advanced Micro Device,2004, "28F020-256K CMOS Flash Memory", www.futurlec.com/Memory/28F020pr.shtml.

[3] Barr,M., 2001, "Introduction to Memory Types", www.netrino. com /publications/Glossary/Memory Types.htm.

[4] Barr, M., 2001, "Memory Types", Embedded System Programming, Vol. 10; No.1, pp.103-104.

[5] Campbell, B., 2002, "Understanding Types of Memory: Different Types of Memory \& Their Uses", www.Buymemory.com/memtypes.htm.

[6] Catalyst Semiconductor, Inc., 1992, "2 Megabit CMOS FLASH MEMORY", Data-Net 2.0.

[7] Douglas, V. H., 1988, "Microprocessors and InterfacingProgramming and Hardware" .McGraw-Hill.

[8] HARRIS Semiconductor, 1992, “CD4052MS CMOS Analog Multiplexer/Demultiplexer”, Data-Net 2.0.

[9] IBM Personal computer Company, 1997, "Types of Memory", www.computekcomputing.com/pdf/xbmemtype.pdf.

[10] Intel Corporation, 1994, "Flash Memory", www.Citeseer. ist. psu. edu /context/1257710.

[11] Intel, 1994, "28F020-2048k(256k*8) CMOS FLASH MEMORY", Data-Net 2.0.

[12] Intel, 1995, "28F020 2048k (256x8) FLASH MEMORY”, $\underline{\text { www. }}$ lyring.com/DocumentosLyR/EXT/ICs/Memory/2MbitFlashEPROM. PDF.

[13] Jeff R.,2000, "SST39SF020 flash used for BIOS”, www. iopener. home.fastolfe.net/ihack/0048.htm.

[14] Morris, M. M., Charles R. Kime, 2001, "Logic and computer design Fundamentals", Second Edition Updated, Printice Hall.

[15] Slone, A., 1999, “Computer Communication”, McGraw-Hill. 
[16] SST, 1998, “2Megabit (256x8) Multipurpose Flash”, www. katalogi. iele. polsl.gliwice.p1/upload/39SF020.pdf.

[17] Tyson, J.,2004, "How Rom works", www. howstuffworks. com/ com /.htm. 